\title{
Modelling of creep rupture of ferritic/austenitic dissimilar weld interfaces under mode I fracture
}

\author{
Jia-nan $\mathrm{Hu}^{\mathrm{a}, \mathrm{b}, 1}$, Takuya Fukahoric, Toshihide Igaric, \\ Yasuharu Chuman ${ }^{\mathrm{c}}$, Alan C.F. Cocks ${ }^{\mathrm{a}}$ \\ ${ }^{a}$ Department of Engineering Science, University of Oxford, Parks Road, OX1 3PJ, UK \\ ${ }^{b}$ Sente Software Ltd, Surrey Technology Centre, Guildford, GU2 7YG, UK \\ ${ }^{c}$ Research \& Innovation Centre, Mitsubishi Heavy Industry. Ltd, 717-1, Fukahori-machi, 5-chome, Nagasaki, 851-0392, Japan
}

\begin{abstract}
Dissimilar metal welded structures (DMWs) have been used extensively in conventional and nuclear power generation plants. Evaluation of creep rupture properties of DMWs is critical to the structural integrity assessment. Failure of DMWs can occur in the base metal, the heat-affected zone (HAZ), or the dissimilar interface between the two welded materials, depending on the operating stress and temperature. The primary focus of this work is on interface failure in systems consisting of a ferritic steel (P91 or P22) and an Inconel filler material, which has an austenitic structure. A planar damage zone is introduced within a finite element (FE) framework to model the response of the interface. A traction-separation constitutive law with a Kachanov-type damage accumulation relationship is employed to describe the interface response, with the material parameters calibrated against available creep rupture data in which failure occurred at the dissimilar weld interface. It is found that the difference in damage accumulation along the interface of different DMW systems can be attributed to the mismatch in creep properties of the continuum materials either side of the interface. Diversion of the crack path into the HAZ is also captured as a result of damage accumulation in the heat affected zone (HAZ). The relationship between the empirical damage accumulation model and the major microstructural features that are responsible for interface failure is also discussed.
\end{abstract}

Keywords: Dissimilar metal weld; Interface creep rupture; Damage zone model; P91, P22, Inconel

${ }^{1}$ Corresponding author. Tel.: +44(0)1483685478. E-mail address: jianan.hu@ sentesoftware.co.uk 
Nomenclature

\begin{tabular}{|c|c|}
\hline$\alpha$ & Coefficient of thermal expansion (CTE) \\
\hline$\varepsilon_{i j}^{e}$ & Elastic strain of continuum material \\
\hline$\varepsilon_{i j}^{c r}$ & Creep strain of continuum material \\
\hline$\varepsilon_{i j}^{t h}$ & Thermal strain of continuum material \\
\hline$\dot{\varepsilon}_{0}\left(\dot{\varepsilon}_{0 H}\right)$ & Strain rate in the base metal (heat affected zone) at a reference stress $\sigma_{0}$ \\
\hline$n$ & Power-law creep stress exponent of continuum material \\
\hline$h$ & Softening constant for heat affected zone \\
\hline$b$ & Nucleation constant for heat affected zone \\
\hline$N$ & Number of nucleated cavities in heat affected zone \\
\hline$X$ & Triaxiality factor \\
\hline$\delta_{n}\left(\delta_{t}\right)$ & Normal (tangential) separation of interface zone \\
\hline$T_{n}\left(T_{t}\right)$ & Normal (tangential) traction of interface zone \\
\hline$a$ & Elastic constant of interface zone \\
\hline$\dot{\delta}_{0}$ & Creep separation rate of interface zone at a reference traction $T_{0}$ \\
\hline$\delta_{c}$ & Critical crack opening separation \\
\hline$\delta_{n}^{c r}$ & Normal separation of interface zone resulted from creep \\
\hline$\omega$ & Damage variable of interface zone \\
\hline$m$ & Power-law creep constant of interface zone \\
\hline
\end{tabular}




\section{Introduction}

Dissimilar metal welded joints (DMWs) are used extensively in many high temperature engineering applications in which different materials are used in different regions of a plant to satisfy the mechanical, environmental and/or economical requirements of the design [1]. In conventional or nuclear power generating plant, DMWs between ferritic low alloy steels and austenitic stainless steels or between different grades of ferritic steels are commonly used [2-4]. Due to the thermo-mechanical mismatch between the two materials, filler metals (such as Inconel) are often used and buttered on one material before being joined to the other. This, ensures the mutual solubility between the two welded materials, and also helps mitigate against the residual stresses that can be produced when the DMWs are heated to elevated temperatures in service $[3,4]$.

Evaluation of the creep rupture properties of DMWs is critical to the structural integrity assessment. A variety of failure modes associated with DMWs have been identified during monotonic uniaxial creep rupture tests of planar components or solid pipes [3, 5, 6]. There are three major categories of failure (Fig. 1), namely: base metal failure (Type V); heat affected zone (HAZ) failure (Type IV); and failure at the interface between one metal and the filler metal (Type VI or VII), whereby Type VII refers to failure along the interface, and Type VI failure initiates where the interface meets the free surface, but eventually diverts into the HAZ or even the base metal. Similar crack path changes have also been observed in similar metal welded (SMW) components [7, 8] which have been simulated by examining creep crack growth (CCG) in SMW models containing an initial crack at different positions [712]. These studies demonstrate that, the effect of mismatch in creep properties surrounding the crack, applied load level, initial crack position, specimen geometric constraint and even the width of the HAZ etc, can all contribute to the crack path change by promoting the initiation and propagation of a secondary crack in the soft material to the side of the initial crack [7,8]. These factors may help explain the Type VI failure mode shown in Fig. 1 for DMW components, but studies on intact DMW components without an initial defect have not been extensively conducted. Moreover, changes in environmental conditions such as the operating temperature and applied stress level may lead to a change of the dominant failure mode. A comprehensive understanding of the DMW failure mechanism and when a given mechanism dominates is yet to be achieved.

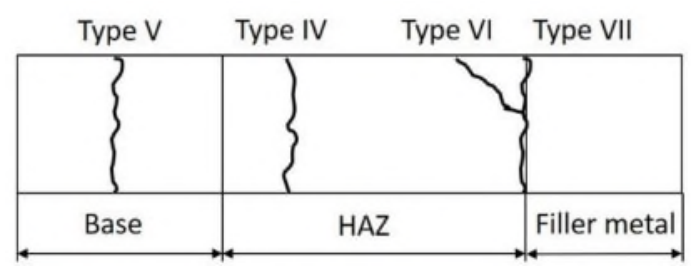

Fig. 1. Typical failure modes associated with dissimilar metal welded systems

In this work we are interested in the creep failure at dissimilar welded interfaces when one metal is ferritic $9 \mathrm{Cr}-1 \mathrm{MoVNb}$ (designated P91) steel or 2.25Cr-1Mo (designated P22) and the filler metal is an austenitic Inconel alloy (designated Inco). Monotonic uniaxial creep rupture tests of different DMWs have been carried out by different research groups at $550^{\circ} \mathrm{C}[3,5,6]$. Laha et al [3] adopted Inco182 as the filler metal, while other researchers [5, 6] employed Inco82. Fig. 2 summarizes the results of experiments on P91/Inco182/Alloy 800 and P22/Inco182/Alloy 800 DMWs conducted by Laha et al [3] and P91/Inco82/SUS304 conducted by Yamazaki and Yamashita et al [5, 6]. Results of creep tests on the pure base metals of P91 or P22 and the trend lines for all the data obtained by Laha et al [3] are also shown in the figure. At most applied stresses, DMWs are found to be weaker than the counterpart base metals. Also P22 DMWs are found to be weaker than P91 DMWs in all these uniaxial tests. In the double logarithmic plot of Fig. 2, a transition of slope of the trend lines can be clearly seen when the mode changes to interface failure, which dominates at relatively low stresses and long times to failure, and has occurred predominantly at the ferritic/austenitic interface, despite the use of different Inconel fillers. Note that the interface failure life of the P91 DMWs tested 
by the different investigators is observed to be similar within the tested stress range. Further, metallurgical observations of the tested samples have revealed a sharp transition in microstructure, physical properties and chemical composition across the interface, which leads to discontinuous mechanical properties, such as hardness, and the generation of residual stresses [3]. Elongated precipitates and cavities have also been observed along the interface [3, 4]. However, despite the above experimental findings, very few theoretical and numerical simulation studies have been conducted of damage accumulation and creep rupture along the interface. In addition, steam power generating plants have seen an extensive use of P91/Inco/P22 DMWs, i.e. P91 welded with P22 using Inconel filler metal, whereby in these systems the primary interface failure can occur at either of the ferritic/austenitic interfaces under complex multi-axial and/or cyclic thermo-mechanical loading experienced in service. Detailed theoretical and computational studies are needed to develop a more in-depth understanding of the failure mechanisms, and when a given mechanism dominates, in order for engineers to accurately predict the failure location and assess the creep rupture life of different DMW components.

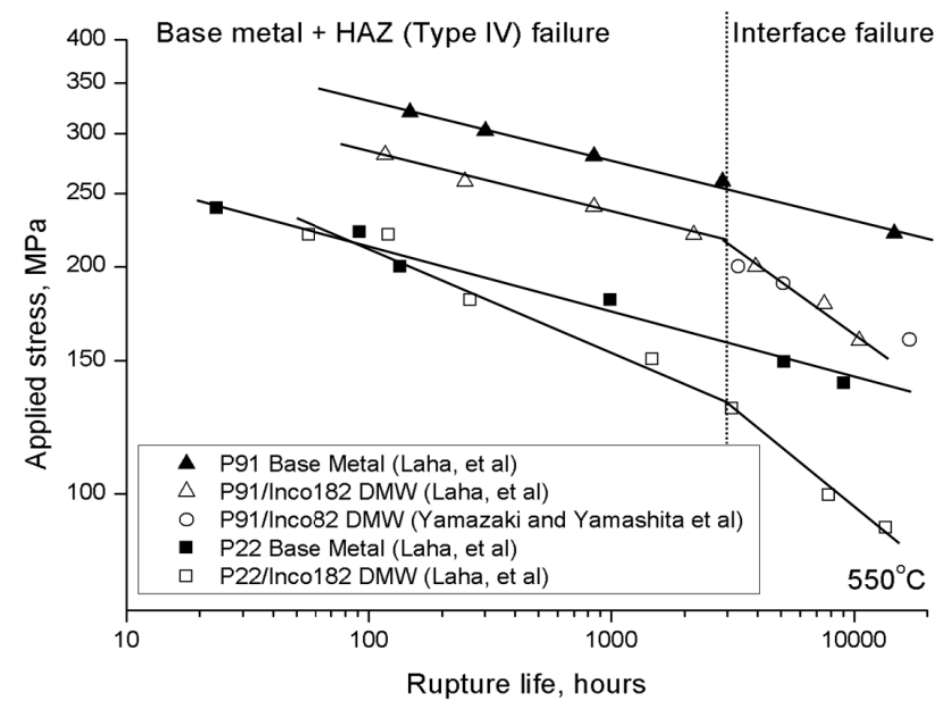

Fig. 2. Comparison of creep rupture lives of P91 (P22) base metals and ferritic/austenitic dissimilar metal welded (DMW) joints P91 (P22) / Inconel at 550 ${ }^{\circ} \mathrm{C}[3,5$, 6]. The solid lines are the trend lines of the tested data in [3].

In this study, we investigate the interface failure of two ferritic/austenitic DMW systems at $550^{\circ} \mathrm{C}$, namely $\mathrm{P} 91 / \mathrm{Inco}$ and P22/Inco system, through an analytical and computational damage zone approach. Such an approach has proved to be robust in capturing the development of damage, describing crack initiation and subsequent propagation in a range of nonlinear fracture processes [13]. We consider the situation where the load is applied perpendicular to the interface, to simulate remote mode I fracture (although mix-mode conditions prevail at the interface due to the mismatch in mechanical and thermal properties). Fracture under remote mixed-mode conditions will be considered in detail elsewhere [14]. An appropriate interface zone model incorporating a Kachanov-type ductility exhaustion damage accumulation law [7-9, 15] is implemented in ABAQUS to simulate and evaluate interface failure of each DMW system. Such interface zone models have been adopted in our previous work [16] on the interface failure of two DMW components with a simple two-dimensional plate geometry, in which the effect of the HAZ was ignored. In this work, the model is improved to include the following aspects: (1) A more practical three-dimensional axisymmetric cylinder structure is used to simulate the geometry of actual test specimens; (2) The softening effect of HAZ is incorporated, with different layers possessing different creep properties, as obtained from the insight given by Laha et al [3] and Li et al [17]; (3) In total four detailed DMW components are considered here. We first take the two DMW components (P91/Inco182, P22/Inco182) tested by Laha et al [3] and recalibrate the parameters against their available interface failure data shown in Fig. 2 for both components. These parameters are subsequently used to evaluate another two DMW components (P91/Inco82, 
P22/Inco82) tested by Yamazaki and Yamashita et al $[5,6]$. Parametric studies such as sensitivity to creep properties and geometric constraint are also examined, taking the P92/Inco82 DMW system as a typical example. Results for different DMW systems from different sources are compared and explained in terms of the relative timescales for crack initiation and propagation. The aim is to probe the intrinsic interface failure mechanism and how it is affected by the creep properties of the surrounding materials. Finally, the relationship between the calibrated parameters and the microstructural features is also discussed.

\section{Modelling of DMW interface creep rupture}

A complete DMW system contains base or parent metal(s), heat-affected zone (HAZ), filler metal (or weld metal), as well as the interface region between the two dissimilar materials. Different parts may possess different creep properties and thus need to be modelled in different ways [3, 18]. Here we are interested in the range of loading conditions identified in Fig. 2 that result in failure along the interface, and we choose constitutive relationships that are appropriate for these situations.

\subsection{Base metal and filler metal}

Both the base and filler metals are considered to be homogeneous and isotropic, with their creep behaviour described by a simple empirical power-law constitutive model, i.e. a steady-state Norton creep law. The transition in slope observed in Fig. 2 indicates that over the range of stress in which interface failure dominates, the base metal would fail at much longer times to failure, and over the time scales of interest has not yet entered the tertiary stage of creep, thus damage development and softening of the base metal can be ignored. The complete multi-axial form of the constitutive response for the total strain rate for the base or filler metals can be written as the sum of the elastic, creep and thermal strain rate tensors:

$$
\dot{\varepsilon}_{i j}=\dot{\varepsilon}_{i j}^{e}+\dot{\varepsilon}_{i j}^{c r}+\dot{\varepsilon}_{i j}^{i h}=\frac{1+v}{E}\left(\dot{\sigma}_{i j}-\frac{v}{1+v} \dot{\sigma}_{k l} \delta_{i j}\right)+\frac{3}{2} \dot{\varepsilon}_{0}\left(\frac{\sigma_{e}}{\sigma_{0}}\right)^{n-1} \frac{s_{i j}}{\sigma_{0}}+\alpha \dot{T}_{t e m p}
$$

where $\sigma_{e}$ and $s_{i j}$ are true effective stress and true deviatoric stress, $E$ and $v$ are Young's modulus and Poisson's ratio, $\delta_{i j}$ is the Kronecker delta, $\dot{\varepsilon}_{0}$ and $\sigma_{0}$ are reference creep strain rate and reference stress respectively, $\alpha$ is the linear coefficient of thermal expansion (CTE, assumed to be isothermal), $\dot{T}_{\text {temp }}$ is the rate of change of temperature and $n$ is the steady state creep exponent. The thermo-elastic constants for the base and filler metals at $550^{\circ} \mathrm{C}$ are listed in Table 1, while the creep constants are shown and compared in Fig. 3 in the plots of creep rates at different loads. Note that the variation of creep properties can be seen between measurements by different researchers even on the same ferritic steel.

\subsection{Heat-affected zone (HAZ)}


The HAZ can be sub-divided into a coarse-grained heat affected zone (CGHAZ), fine-grained heat affected zone (FGHAZ) and inter-critical heat affected zone (ICHAZ) [19] with increasing distance from the filler metal. Type IV failure occurs predominantly in the FGHAZ as a result of strain softening and cavity nucleation and growth under the high local triaxial stress states arising from the local constraint imposed on the deformation of the soft FGHAZ material by the adjacent more creep resistant sections of the HAZ [17, 20-22]. Over the stress range of interest type IV failure competes with interface failure and even under situations where interface failure dominates the crack path can deviate into the HAZ towards the end of life (Type VI failure). The effect of strain softening and cavitation on the constitutive response of the HAZ must now be taken into account. The thermo-elastic part of the model in Eq. (1) is the same as for the ferritic base metal, while the creep part is modified to take into account the effect of strain softening $[23,24]$ :

$$
\dot{\varepsilon}_{i j}^{c r}=\frac{3}{2} \dot{\varepsilon}_{0 H}\left(\frac{\sigma_{e}}{\sigma_{0}}\right)^{n-1} \frac{s_{i j}}{\sigma_{0}} \exp h \bar{\varepsilon}^{c r}
$$

where $h$ is a softening constant and $\bar{\varepsilon}^{c r}$ is the total effective accumulated creep strain. $\dot{\varepsilon}_{0 H}$ is a different reference creep strain rate for the HAZ, not to be confused with that for the base metals in Eq. (1). Eq. (2) demonstrates that the creep rate increases with increasing creep strain. Note that the creep parameters may vary in different zones. In practice, we simply independently model the CGHAZ and FGHAZ in this study. A simple damage accumulation model is employed for each region of the HAZ to characterize the formation of cavities of the order of the grain size developed by Gonzales et al [25] based on the experimental observations of Masuyama and Yamaguchi [26]. They observed the following sequence of processes leading to failure: initially, small sub-micron size cavities initiate at triple points or precipitates at the grain boundaries; these cavities grow and link to completely surround a grain, isolating it from the surrounding matrix, effectively forming a large void of the order of the grain size; these cavities experience limited growth after formation, but as more and more form they eventually coalesce leading to failure. It is found experimentally that failure occurs when the number density of these grain-sized cavities reaches a critical value. The model assumes that failure is nucleation controlled and that the rate of nucleation of the large grain-sized pores is proportional to average rate of nucleation of the small pores that form around the perimeter of a grain; these pores in turn are assumed to nucleate according to a Dyson/Greenwood [27-29] criterion in which the rate of nucleation is proportional to the macroscopic effective strain rate and the square of the stress normal to the grain-boundary. By projecting the stress state onto a unit sphere, Gonzales et al [25] obtain the following relationship for the rate of nucleation of the grain-sized pores

$$
\dot{N}=b \dot{\varepsilon}_{e}^{c r}\left(\frac{4}{9}+\frac{20}{9} X^{2}\right)
$$

where $\dot{N}$ is nucleation rate, $\dot{\varepsilon}_{e}^{c r}$ is effective creep rate, $b$ is a material constant, $\sigma_{m}$ is the hydrostatic component of stress and $X=3 \sigma_{m} / 2 \sigma_{e}$ is the triaxiality factor. Gonzales et al [25] demonstrate that this model provides a good correlation of the distribution of grain-sized cavities in crept notched components of simulated P91 FGHAZ material. Igari et al [21] and Fukahori et al $[21,30]$ have adopted this model for welded components and identified a critical number of cavities $N_{c}=40,000$ for Type IV failure in P91 ferritic steel, based on the measurement of the average grain size in P91 FGHAZ. For P22 ferritic steel, $N_{c}=10,000$ due to a different average grain size from that of P91. In this paper, our main focus is on interface failure, and we do not further model the detailed influence of cavity nucleation on the creep behaviour of HAZ. We simply track the parameter $N / N_{c}$, which is stored as a state variable, to provide an indication of damage development in the HAZ as the interface damages and a crack propagates along the interface. The values of the thermo-elastic, softening and cavity nucleation material parameters in Eqs.(1), (2) and (3) are listed in Table 1 for the different regions of the welded component. Note that for simplicity, the thermo-elastic properties of HAZ are assumed to be the same as the corresponding base metal. Due to a lack of material properties for P22, we use the same values of $h$ and $b$ as for P91. The creep properties of the different weld components are shown and compared in Fig. 
3. Note that both P91/Inco and P22/Inco considered here are creep-hard [8, 11] or over-matched [12] DMW components at moderate stress levels, where the weld metal Inconel is more creep resistant than other components. The FGHAZ is further observed to be much softer than the CGHAZ. A variation of creep properties can be seen between the measurements and simulations undertaken by Laha et al [3], Kawashima et al [31] and Fukahori et al [30]. Laha et al [3] conducted experiments on simulated HAZ material which had microstructures, prior austenitic grain size and hardness equivalent to those of the HAZ of the actual welded joint [32]. While Kawashima et al [31] and Fukahori et al [30] have regarded the CGHAZ to have the same creep properties as the base metal. Here we have also included the effects of strain softening and cavitation.

Table 1. Thermo-elastic constants for base and HAZ materials at $550^{\circ} \mathrm{C}$

\begin{tabular}{|c|c|c|c|c|c|}
\hline \multirow{2}{*}{ Parameter } & \multicolumn{2}{|r|}{ P91 } & \multicolumn{2}{|r|}{ P22 } & \multirow{2}{*}{ Inco82/182 } \\
\hline & base & HAZ & base & HAZ & \\
\hline$E$ (GPa) & \multicolumn{2}{|r|}{174} & \multicolumn{2}{|r|}{169} & 182 \\
\hline$v$ & \multicolumn{2}{|r|}{0.3} & \multicolumn{2}{|r|}{0.3} & 0.3 \\
\hline$\alpha \times 10^{-6} /{ }^{\circ} \mathrm{C}$ & \multicolumn{2}{|r|}{12.5} & \multicolumn{2}{|r|}{14.6} & 15.0 \\
\hline$h$ & & $6.0[25]$ & & 6.0 & \\
\hline$b$ & & $1500[21,30]$ & & 1500 & \\
\hline$N_{c}$ & & $40,000[21,30]$ & & $10,000[21,30]$ & \\
\hline
\end{tabular}

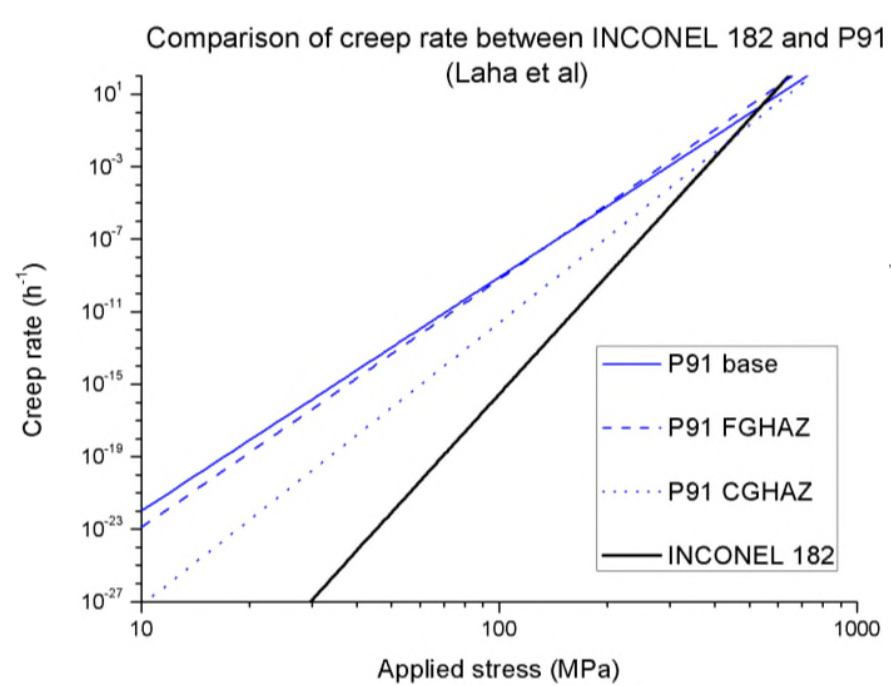

(a)

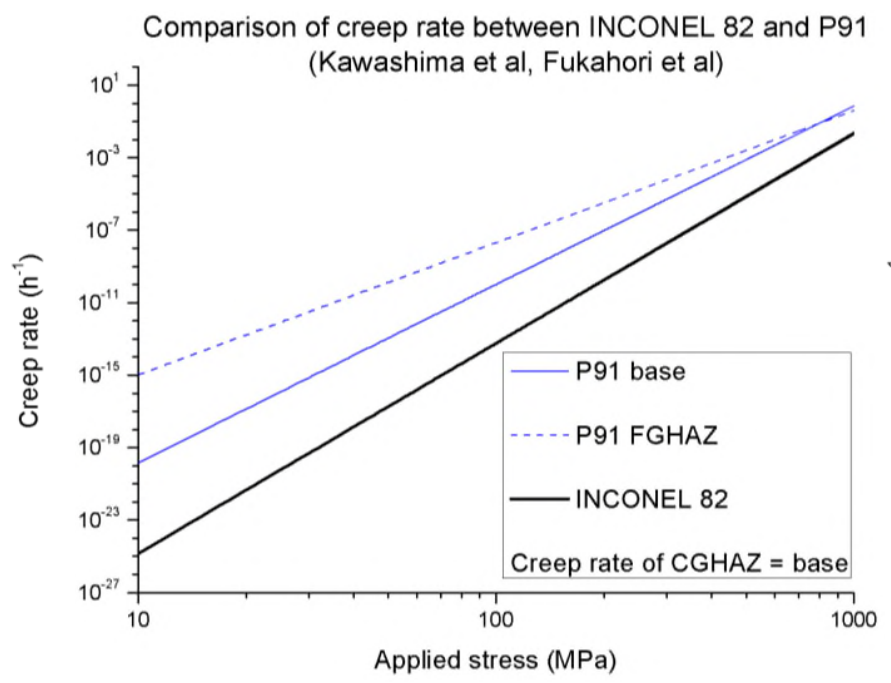

(c)

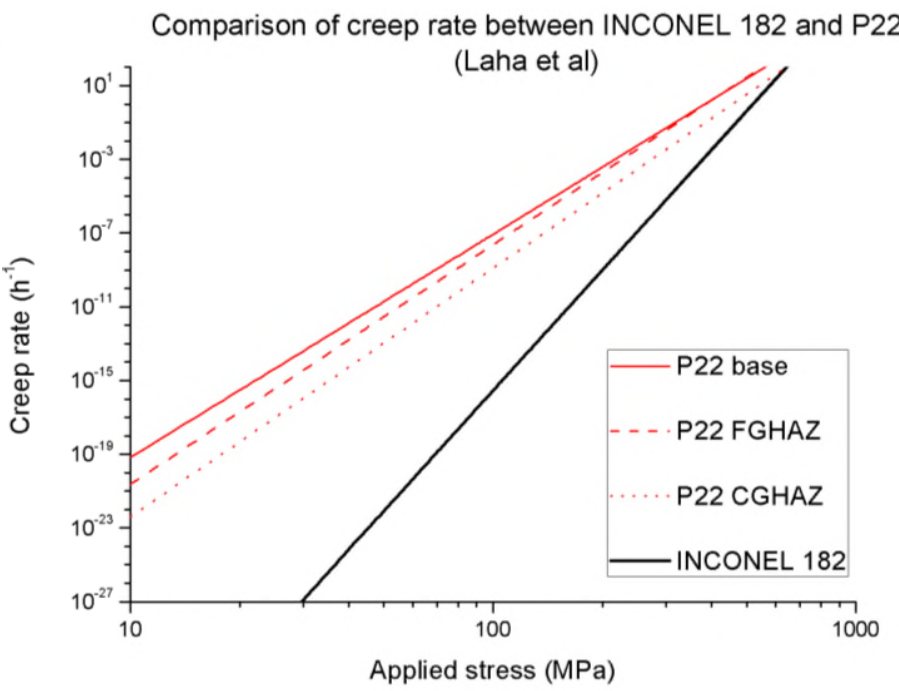

(b)

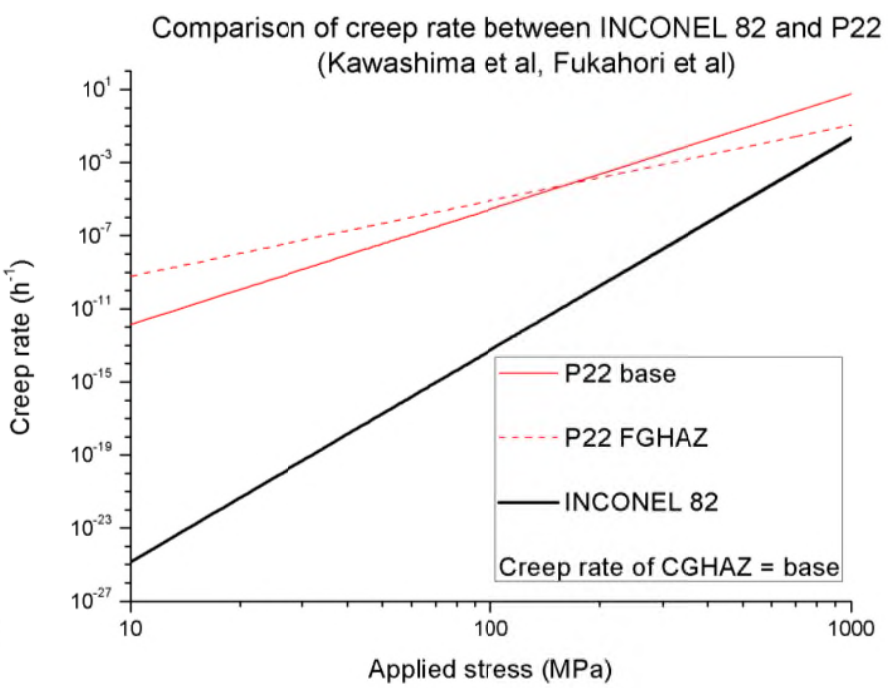

(d) 
Fig. 3. Comparison of creep properties (creep rate at different loads) of base metals, $\mathrm{HAZ}$ and filler metals at $550^{\circ} \mathrm{C}$ measured by Laha et al [3], (a) P91 / Inco182, (b) P22 / Inco182, and by Kawashima et al [31] and Fukahori et al [30], (c) P91 / Inco82, (d) P22 / Inco82. All the figures are plotted in the same scale. Note that reference creep rate of CGHAZ is assumed to be identical to that of base metal in (c) and (d)

\subsection{Interface region}

The interface region of each DMW system is modelled as an ultra-thin layer of interface zone. Deformation and failure of the interface can be characterized by the interface separation or opening behaviour using appropriate traction-separation constitutive relationships. In two-dimensional problems, both tractions and separations have two components, tangential $\left(T_{t}, \delta_{t}\right)$ and normal ( $\left.T_{n}, \delta_{n}\right)$. A detailed description of interface models of this type and how they are implemented can be found in [13, 33]. However, these models, either classified as non-potential-based or potential based models, largely adopt rate-independent traction-separation laws that relate the normal and tangential displacements or displacement jumps $(\delta)$ to the corresponding tractions $(T)$. Here in the current study, we employ a new phenomenological rate-dependent interface zone model, i.e. we relate tractions to the displacement rates (or separation rates $\dot{\delta}$ ) under creep loading. Further, a Kachanov type damage variable $\omega$, is incorporated to reflect the gradual accumulation of damage at the interface zone, as motivated by the classical continuum damage mechanics approach originally proposed by Kachanov [34]

$$
\begin{aligned}
& \dot{\delta}_{n}=\dot{\delta}_{n}^{e}+\dot{\delta}_{n}^{c r}=\frac{a \dot{T}_{n}}{1-\omega}+\dot{\bar{\delta}}_{0}\left(\frac{T_{n}}{T_{0}(1-\omega)}\right)^{m} \\
& \dot{\delta}_{t}=\dot{\delta}_{t}^{e}+\dot{\delta}_{t}^{c r}=\frac{a \dot{T}_{t}}{1-\omega}+\dot{\delta}_{0}\left(\frac{T_{t}}{T_{0}(1-\omega)}\right)^{m}
\end{aligned}
$$

where all the subscripts " $n$ " and " $t$ " refer to normal and tangential directions and all the superscripts " $e$ " and " $c r$ " refer to elastic and creep contributions. $a$ and $m$ are material constants, where $m$ may be different to the exponent $n$ in the power-law creep models of the base and HAZ materials (Eqs.1 and 2). $\dot{\delta}_{0}, T_{0}$ are reference separation rate and reference traction. In order to limit the number of material parameters we assume that the reference separation rate is the same in the normal and tangential directions.

We assume that damage accumulation in the interface zone is only related to the normal creep separation $\delta_{n}^{c r}$, and that failure occurs when $\delta_{n}^{c r}$ reaches a critical separation $\delta_{c}$. We further assume that

$$
\omega=\delta_{n}^{c r} / \delta_{c}
$$

The use of a displacement-based damage criterion of this type resembles the strain-based creep ductility exhaustion model frequently used for creep crack growth $[7-10,12,15]$. This type of model parallels the conventional continuum damage mechanics stress-based damage law proposed by Kachanov-Rabotnov [34, 35] but is simpler and contains fewer fitting parameters [15]. In addition, use of the damage variable $\omega$ in both the elastic and creep parts in Eqs. (4a) and (4b) provides a characterization of the degradation of interface stiffness and rate dependent properties as the damage gradually accumulates at this interface zone. The effect of using different types of damage model with the structure of Eq. (4) on creep crack growth is evaluated in [36]. We also explore the coupling between the normal and tangential response using a physically based interface cavitation model [14].

\section{Finite element analysis framework}

\subsection{Dissimilar metal welded solid cylinder (axisymmetric problem)}

Here we explore how the proposed empirical Kachanov-type damage model described above can be used to capture and simulate interface failure in DMW systems through numerical simulation using the commercial finite element code ABAQUS. 
Only fracture under remote mode I loading at $550^{\circ} \mathrm{C}$ at a dissimilar interface is considered in this section (note: due to material mismatch, mixed mode conditions exist at the interface - we do not evaluate the degree of mode mixity here, but simply examine the effect of material combinations on the global macroscopic response). Interface creep rupture data obtained by Laha et al [3] (Fig. 2) is used to calibrate the model. Accordingly, an axisymmetric solid cylinder model is established as shown in Fig. 4(a) to simulate the geometry of the actual specimen, with the specimen gauge length and diameter as well as the dimensions of FG(CG)HAZ following the experiment conducted by Laha et al [3]. The model is comprised of two materials (ferritic steel and Inconel) with one ferritic-austenitic dissimilar interface, as simulated by a thin layer of interface elements (described in section 3.2) inserted between the CGHAZ of the ferritic steel and Inconel. Interfaces between P91/Inconel and between P22/Inconel are investigated independently. The applied uniaxial load direction is perpendicular to the HAZ and interface. Boundary conditions are also shown in the figure. A mesh dependence analysis has been carried out by Hu et al [16], where different numbers of interface elements have been adopted, which are equally distributed along the interface zone. The results show that the creep crack initiation time and creep crack growth rate both decrease with decreasing mesh size along the dissimilar interface, but the sensitivity becomes negligible as the mesh size decreases to $30 \mu \mathrm{m}$. Therefore, 100 interface elements were used with interface elements of size $25 \mu \mathrm{m}$. Meshes in the HAZ and Inconel are refined to provide the finest elements adjacent to the interface zone (Fig. 4b).

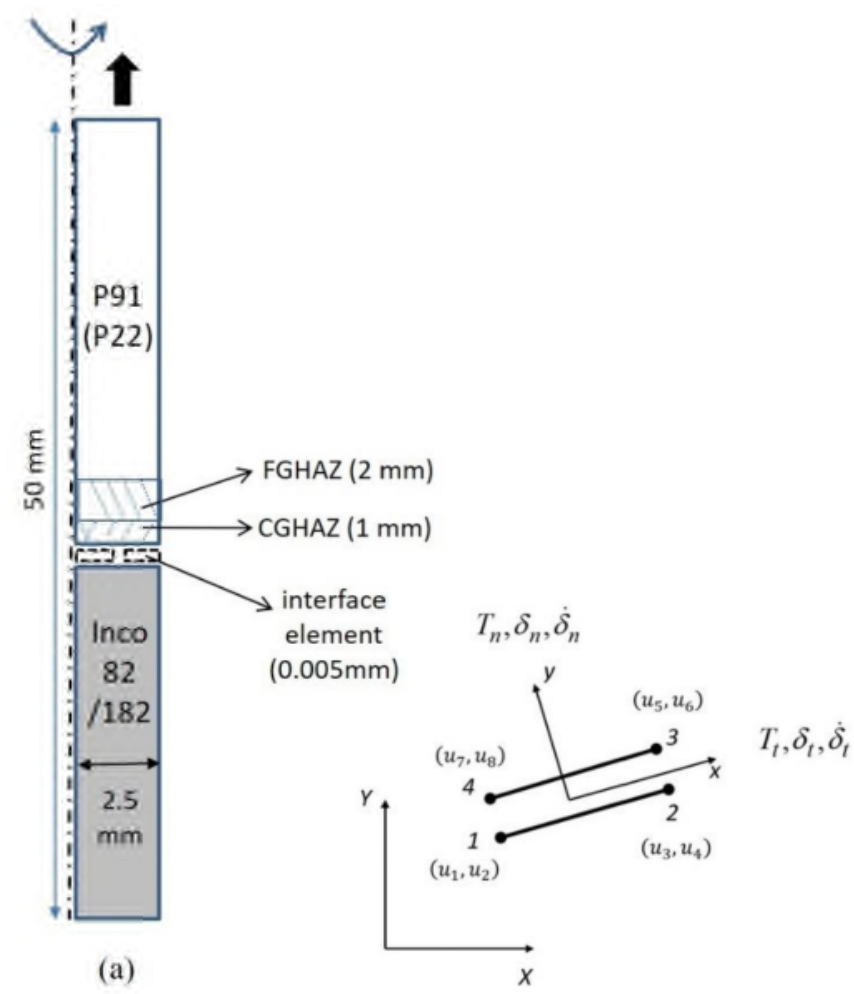

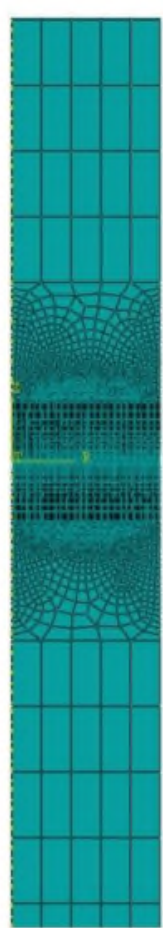

(b)

Fig. 4. Schematic of axisymmetric solid cylinder rod models of (a) P91(P22) / Inco182(82) DMW system subject to uniaxial creep loading at $550^{\circ} \mathrm{C}$. FGHAZ and CGHAZ are created as parallel to the interface and perpendicular to the direction of the applied load. The interface is modelled as a zone filled with four-node linear interface elements, each node having two degrees of freedom (normal and tangential). (b) Refined meshes around and towards the interface (e.g. in CGHAZ)

\subsection{Linear interface element}

The interface element consists of two lines and four nodes with a linear displacement formulation (Fig. 4a). The two lines connect the faces of adjacent bulk elements, i.e. they share the same surface and nodes. In the current computation, the order of the nodes of an interface element is counterclockwise. Each node has two degrees of freedom (normal and tangential) and each interface element has a total of eight nodal displacements $(u)$ and corresponding displacement increments $(\Delta u)$. The element is coded as an ABAQUS User-defined Element Library Subroutine (UEL), as described in the Appendix. At the beginning of a 
simulation, the thickness of interface layer is zero, but the two surfaces of each interface element separate as the adjacent bulk elements deform in different rates. Transformation of local coordinates or rotation of interface elements is taken into account in the simulation $[13,37]$.

\subsection{Computational scheme}

In the simulation, for the continuum elements of the base and filler metals, we employ the in-built thermo-mechanical model (Eq. 1) provided by ABAQUS. For the continuum elements of the FG(CG)HAZ, while the thermo-elastic properties are given by ABAQUS, the power-law creep model with softening (Eq. 2) and the cavity nucleation model (Eq. 3) are implemented using a CREEP subroutine. The number ratio of cavities $\left(N / N_{c}\right)$ is stored as a solution dependent state variable in the subroutine. For the interface elements, the rate-dependent traction-separation law (Eq. 4) and Kachanov-type damage variable is implemented in the ABAQUS User-defined Element Library Subroutine (UEL). Axisymmetric quadrilateral elements (CPX4) are used for all the continuum elements, which are compatible with the shape functions employed in the newly developed interface elements. The integration area of each individual axisymmetric interface element is calculated by $\int 2 \pi r d r d \theta$, where $r$ is the distance from the centre of the interface element to the axisymmetric axis.

Two steps are used in the simulation, with the first one being STATIC elastic loading and heating $\left(550^{\circ} \mathrm{C}\right)$, and the last one being VISCO creep deformation. Loads are selected based on the creep rupture data shown in Fig. 2. In the VISCO creep step, the FE analysis requires global convergence in the solution of stress and strain in the continuum elements as well as local convergence in the solution of tractions and separations in the interface elements. At the beginning of each time increment, ABAQUS provides UEL with the current time increment $(\Delta t)$, nodal displacement and displacement increment, which determines the normal and tangential separation increments $\left(\Delta \delta_{n}, \Delta \delta_{t}\right)$. The normal and tangential tractions $\left(T_{n}, T_{t}\right)$ from the last increment are stored as state variables in the UEL, and specification of the evolution relationships $\partial T / \partial \delta$ are derived from Eq. (4) (see the Appendix). Based on these, the change of normal and tangential tractions $\left(\Delta T_{n}, \Delta T_{t}\right)$ over the increment and the values at the end of the increment $\left(T_{n}+\Delta T_{n}, T_{t}+\Delta T_{t}\right)$ are calculated through a Newton-Raphson iteration scheme. Details of the Newton-Raphson scheme can be found in the Appendix.

\section{Simulation of creep rupture at dissimilar interface}

\subsection{Calibration of creep properties of interface zone}

Following the procedure described in our previous work [16], here we first calibrate the creep properties of the interface zone based on the interface creep rupture data in Fig. 2. We divide the calibration process into two steps (analytical and numerical). In step 1, it is assumed that the normal traction $T_{n}$ along the interface is uniform and equal to the applied uniaxial stress. The damage accumulation rate $\dot{\omega}$ is then uniform for each interface element and all the elements fail at the same time. Considering Eqs. (4a) and (5), $\dot{\omega}$ in each interface element can be written as

$$
\dot{\omega}=\frac{\dot{\delta}_{n}^{c r}}{\delta_{c}}=\frac{\dot{\delta}_{0}}{\delta_{c}} \cdot\left(\frac{T_{n}}{T_{0}(1-\omega)}\right)^{m}
$$

The time to failure at a given applied load can then be obtained by directly rearranging and integrating Eq. (6), noting that $\omega=0$ at time $t=0$ and 1 when $t=t_{f}$. Then 


$$
\int_{0}^{1}(1-\omega)^{m} d \omega=\int_{0}^{t_{f}} \frac{\dot{\delta}_{0}}{\delta_{c}} \cdot\left(\frac{T_{n}}{T_{0}}\right)^{m} d t \rightarrow \frac{1}{m+1}=\frac{\dot{\delta}_{\hat{0}}}{\delta_{c}} \cdot\left(\frac{T_{n}}{T_{0}}\right)^{m} t_{f}
$$

Using a double logarithmic scale, Eq. (7) can be rewritten as

$$
\log \left(\frac{T_{n}}{T_{0}}\right)=-\frac{1}{m} \log t_{f}+\frac{1}{m} \cdot \log \left(\frac{\delta_{c}}{\dot{\delta}_{0}} \cdot \frac{1}{m+1}\right)
$$

Assuming the reference traction lies within the applied stress range of the experimental data (e.g. $T_{0}=200 \mathrm{MPa}$ for P91/Inconel DMW and $T_{0}=100 \mathrm{MPa}$ for P22/Inconel DMW), the two independent parameters, $\delta_{c} / \dot{\delta}_{0}$ and $m$, can be directly obtained by fitting the interface failure data for each DMW system (Fig. 2). The fitted result is summarized in Table 2.

Table 2. Creep properties directly fitted from the interface creep rupture data in Fig. 2

\begin{tabular}{ccc}
\hline DMW system & $\boldsymbol{m}$ & $\delta_{c} / \delta_{0}(\mathbf{h})$ \\
\hline P91/Inconel & 4.48 & $2.2 \times 10^{4}$ \\
P22/Inconel & 3.93 & $4.2 \times 10^{4}$ \\
\hline
\end{tabular}

In practice the normal traction at the interface is not uniform due to elastic, thermo- and creep mismatch between the adjacent continuum materials surrounding the interface. Accordingly, stress can be elevated and concentrated where the interface meets the free surface. Thus damage grows preferentially and cracks initiate in these regions and propagate gradually along the interface until the specimen fails. Therefore, in step 2, we simulate this process in ABAQUS and systematically adjust $\delta_{c} / \dot{\delta}_{0}$, keeping $m$ constant, until the simulations match the experimental data. The DMW systems adopted by Laha et al [3] (P91/Inco182 and P22/Inco182) are used for the calibration, with the creep properties extracted from Fig. 3 (a) and (b). Then we predict and evaluate the creep rupture life of the DMW systems adopted by Yamazaki et al and Yamashita et al $[5,6]$ using the same calibrated parameters. For simplicity, elastic constants in Eq. (4) remain the same as used in our previous study $\left(a=1 \times 10^{-6} \mathrm{~mm} / \mathrm{MPa}\right)$ [16]. $\dot{\delta}_{0}$ is chosen such that the material length scale $\dot{\delta}_{0} / \dot{\varepsilon}_{0 H}$ is finite, enabling the singularity of tractions where the interface meets the free surface be eliminated, and so that the separation at the interface is dominated by creep rather than elasticity. It is further found that, the creep rupture life depends mainly on the ratio of $\delta_{c} / \dot{\delta}_{0}$ rather than the detailed values of $\delta_{c}$ and $\dot{\delta}_{0}$, thus the magnitude of $\delta_{c}$ has been simply chosen to be $0.01 \mathrm{~mm}$.

Fig. 5 shows the simulated and predicted total creep rupture life of each DMW system. The newly calibrated interface zone parameters are also shown in the figure. The calibrated value of $\delta_{c} / \dot{\delta}_{0}$ at the end of step 2 is around $50 \%$ higher than that computed in step 1 for the P91/Inco182 DMW system and 13\% lower for the P22/Inco182 DMW system. Comparing data from different sources it is further noticed that, the predicted creep rupture life of P91/Inco82 is close to that of P91/Inco182, consistent with the tested data, while the predicted life of P22/Inco82 is almost a half that of P22/Inco182. Such a trend can be linked to the creep properties mismatch between the two sides of the interface, as illustrated below. 


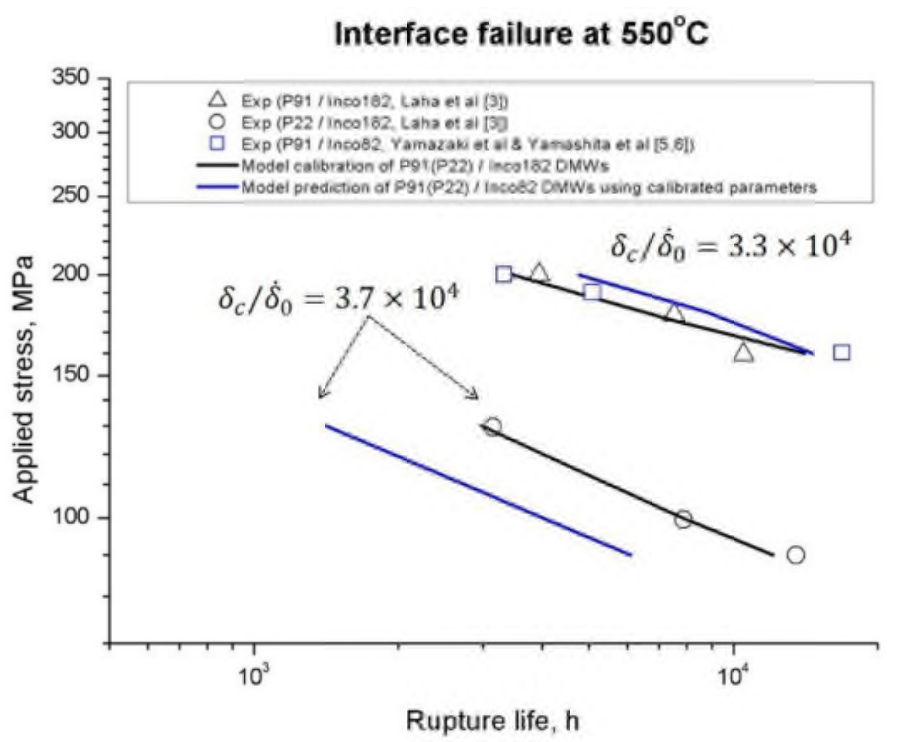

Fig. 5. Experimental and simulated (axisymmetric model) creep rupture life of each DMW system (P91(P22)/ Inco182(82)) under interface failure mode at 550 ${ }^{\circ} \mathrm{C}$. Hollow points are data and solid lines are model calibrated or predicted results. The fitted value of the ratio $\delta_{c} / \dot{\delta}_{0}$ is shown in the figure.

Taking one tested load from each of the P91 DMWs (160 MPa) and P22 DMWs (130 MPa) as an example, the detailed creep damage evolution along the interface of each system is quantitatively illustrated in Fig. 6. Cracks are observed to initiate from the free edge where the interface meets the free surface and gradually propagate towards the center, which is a direct reflection of the stress concentration or enhancement of normal traction at the free edge as a result of physical property mismatch between the materials on the two sides of the interface. Once the crack has propagated across about a quarter of the width of the interface, the rate of propagation increases substantially and extremely small time steps are required for the solution to converge at each increment and to capture the full details of the crack growth process up to final separation. The overall time to failure, is however, well approximated by the time at which the simulations are allowed to terminate, which corresponds to a time increment of $1 \times 10^{-8}$ $\mathrm{h}$ to achieve convergence. It is further noted that, most of the life is spent initiating a crack in most systems (>95\% of total life) and the remaining life for crack propagation is very short (cracks propagate along a heavily damaged interface). However, an exception is observed for the P22/Inco82 DMWs (Fig. 6d), where a crack initiates at a relatively earlier stage (at $\sim 75 \%$ of total life) and the crack propagates slower along a less heavily damaged interface. Comparing between P91/Inco182 and P91/Inco82, the crack is found to initiate slightly later in P91/Inco82 (at 14,400 h) than in P91/Inco182 (at $\sim 13,900$ h), but both systems have a similar crack propagation period. Similarities between the two P91 DMW systems tested by different researchers can be attributed to a similar mismatch in creep properties (difference in creep rates at a given stress) between P91 CGHAZ and Inconel within the range of the applied stresses (Fig. 3 a and c, e.g. $\sim 10^{-8} / \mathrm{s}$ at $160 \mathrm{MPa}$ ). However, it is evident that the creep mismatch between P22 CGHAZ and Inconel measured by Kawashima et al and Fukahori et al [30, 31] is more pronounced (e.g. $\sim 10^{-6}$ at $\left.130 \mathrm{MPa}\right)$ than that measured by Laha et al [3] (e.g. $\sim 10^{-8}$ at $130 \mathrm{MPa}$ ) within the range of the applied stresses (Fig. $3 \mathrm{~b}$ and d). The relatively earlier crack initiation (or faster damage accumulation rate where the interface meets the free surface) in P22/Inco82 DMWs is thus caused by a higher stress (traction) concentration at the free edge as a result of the larger creep mismatch.

The much longer life for crack initiation than that for crack propagation in all the tested systems at each applied stress seems to indicate that the crack growth assessment before a DMW component completely fails is unreasonable for practical applications. However, care needs to be taken when interpreting the simulated creep rupture life from the engineering point of view. While the detailed ratio of crack initiation and propagation depends on the damage accumulation and localized stress state (Eq. 6), one important geometric feature that can contribute to the late crack initiation is the topology of the interface. Here we have adopted the idealization that the interface is flat as shown in Fig. 4. The stress only concentrates around the free edge at the outside surface, which is also the site for crack initiation. However, the actual welded interface would not be flat but wavy, and the surface might 
be irregular, in which case the stress would concentrate at a number of positions along the interface, leading to earlier nucleation at multiple sites. Also, the crack path would become tortuous. The time for crack propagation could then dominate the life of the component. While the actual wavy interface can be modeled and simulated, the idealized flat interface can provide insight and fundamental understanding of the creep crack initiation and propagation processes.

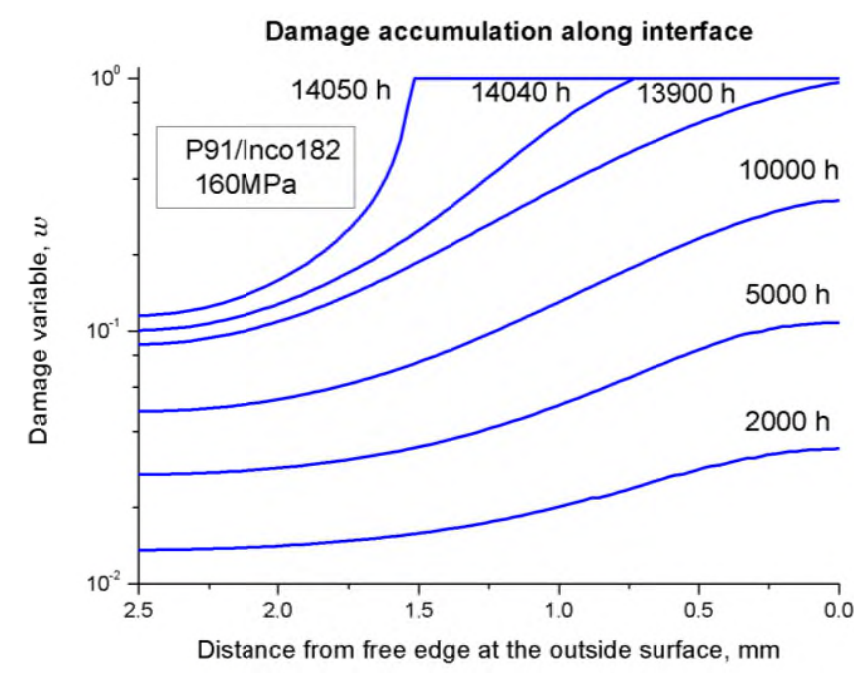

(a)

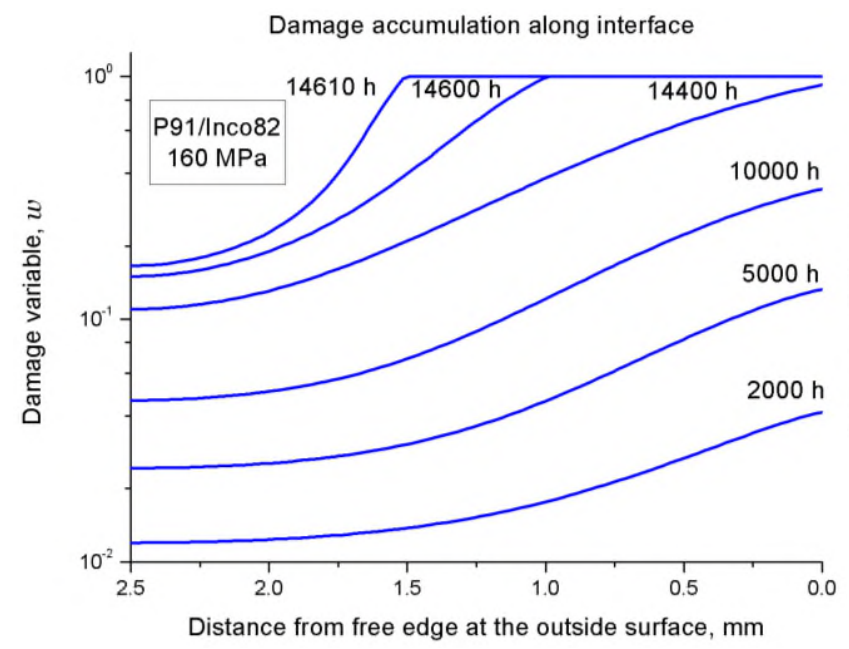

(c)

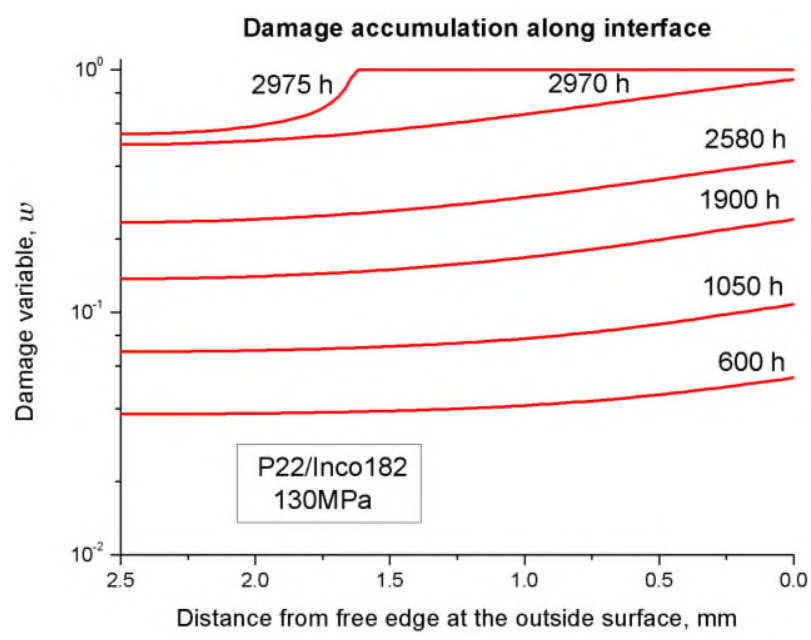

(b)

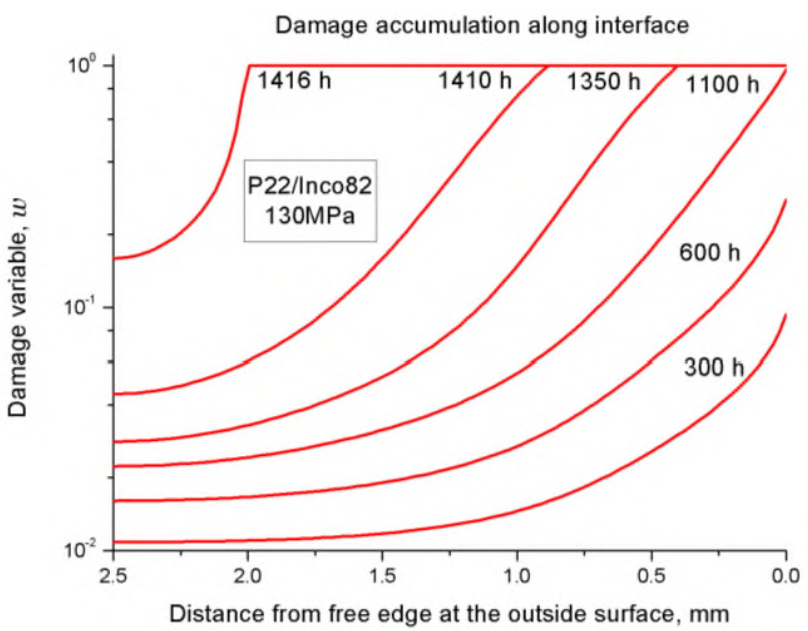

(d)

Fig. 6. Comparison of simulated damage evolution along the interface of (a) P91/Inco182 and (b) P22/Inco182 DMW systems; (c) P91/Inco82 and (d) P22/Inco82 systems. One example stress is chosen for each DMW system.

Further, crack initiation and propagation can be visualized by the evolution of axial stress (perpendicular to the interface) at different stages of creep, as shown in Fig. 7, taking the P91/Inco82 DMW system at $160 \mathrm{MPa}$ as an example. Patterns at other applied stresses as well as other DMW systems are similar. Black crosses in these figures refer to the interface elements. Different stages of behaviour include: pure thermo-elastic loading before creep (Fig. 7a); crack initiation (Fig. 7b); onset of fast crack propagation (Fig. 7c), and final stage of creep rupture (Fig. 7d). Stress is observed to become more and more concentrated at the crack tip as the crack propagates. 


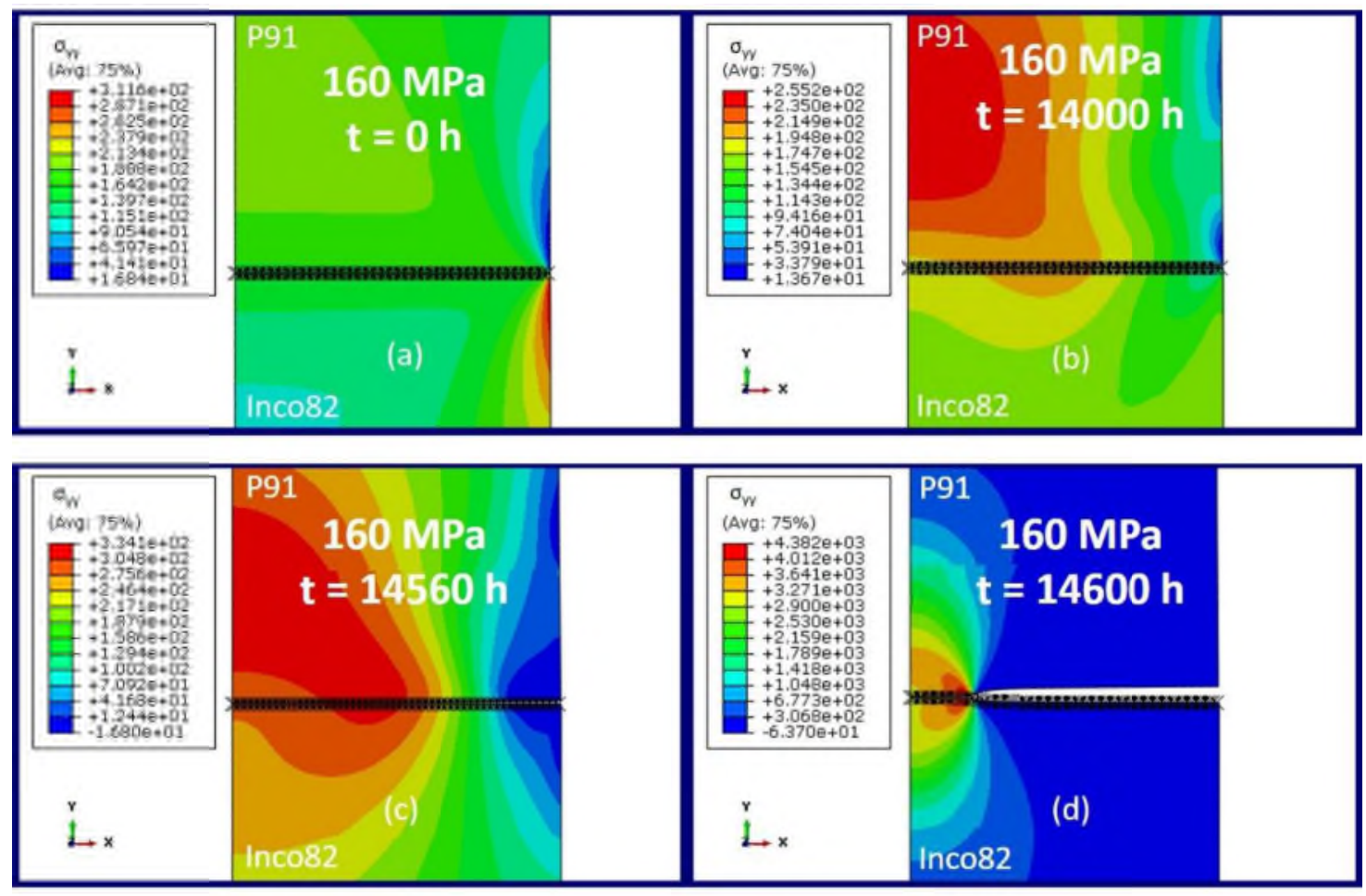

Fig. 7. Axial stress distribution around interface of P91/Inco82 system subjected to a remote applied stress of $\mathbf{1 6 0}$ MPa at different creep rupture stages: (a) before creep; (b) at crack initiation; (c) crack propagation and (d) final stage of creep rupture.

Following the same stages in Fig. 7, the evolution of the normalized cavity density $N / N_{c}$ (Eq. 3) in the HAZ of P91/Inco82 DMW is presented in Fig. 8. For a comparison, equivalent result for the P22/Inco82 DMW is presented in Fig. 9. In both systems, the number ratio in the FGHAZ and CGHAZ increases with time as the creep strain accumulates, with the accumulation rate much faster in the FGHAZ than the CGHAZ (Fig. 8 b\&c and Fig. 9 b\&c). This agrees with the findings that the FGHAZ is the softest region and is the most favorable site for Type IV failure [20-22]. Note that $N / N_{c}$ accumulates $\sim 1-2$ orders of magnitude faster in the FGHAZ of P22/Inco82 compared with that in the FGHAZ of P91/Inco82, partly due to the lower critical cavity density $\left(N_{c}\right)$ in P22 (Table 1) and higher stress triaxiality in the softer P22 FGHAZ compared to the P91 FGHAZ (Fig. 3). Thus P22/Inco82 is more prone to Type IV failure than P91/Inco82. Once the creep crack initiates where the interface meets the free surface, the stress concentration leads to localized creep cavity accumulation around the crack tip in the CGHAZ (Fig. 8 c\&d and Fig. 9 c\&d). An important feature is noted that when the normalized cavity density exceeds unity the crack could divert into the HAZ, i.e. indicating a transition from a Type VII to a Type IV crack path, giving an overall Type VI failure process as discussed in the Introduction and illustrated in Fig. 1. Here we do not further explore the quantitative relationship between Type VI failure and cavity concentration, but emphasize that taking into account the evolution of $N / N_{c}$, the diversion of the crack path into the HAZ for both DMW systems would only occur at the very late stage of creep when the stress concentration is very high and takes only a minimal period that would not significantly affect the total rupture life. This is similar to the observation in creep crack growth (CCG) studies of similar metal welds that the rupture life is mainly determined by the initiation time of the secondary crack [8].

Finally, it is envisaged that, the simulated total creep rupture life is relatively independent of the dimension of the model because of the predicted short period of crack propagation in these models. However, as mentioned before, the model has employed an idealized geometry of an interface, and the geometric size effect could become more important in practical irregular interfaces. 

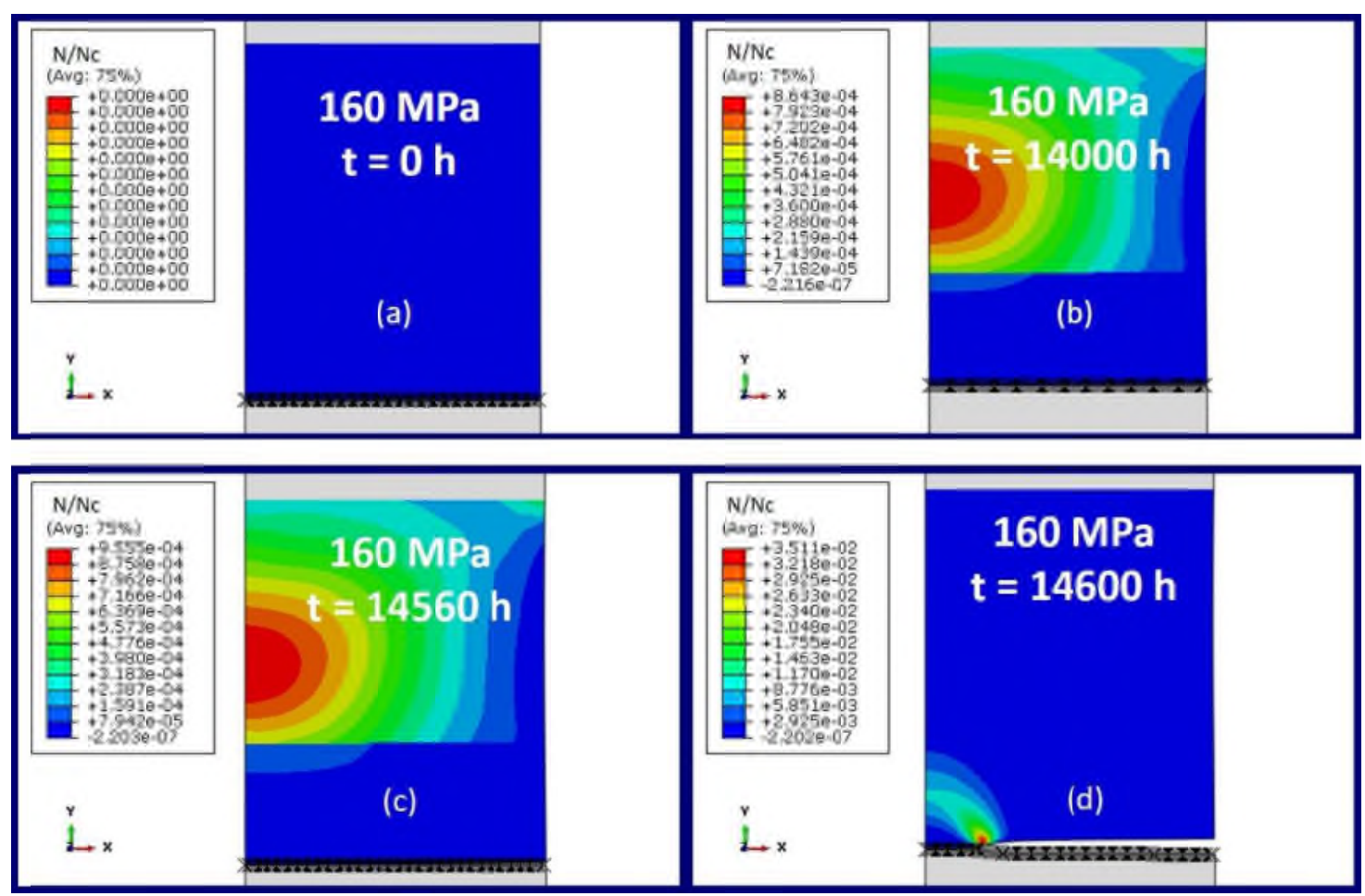

Fig. 8. Evolution of the solution dependent variable $N / N_{c}$ (number ratio of creep cavities Eq. 3) in HAZ of P91/Inco82 DMW system, subjected to a remote applied stress of $160 \mathrm{MPa}$, at different creep rupture stages: (a) before creep; (b) at crack initiation; (c) crack propagation and (d) final stage of creep rupture.
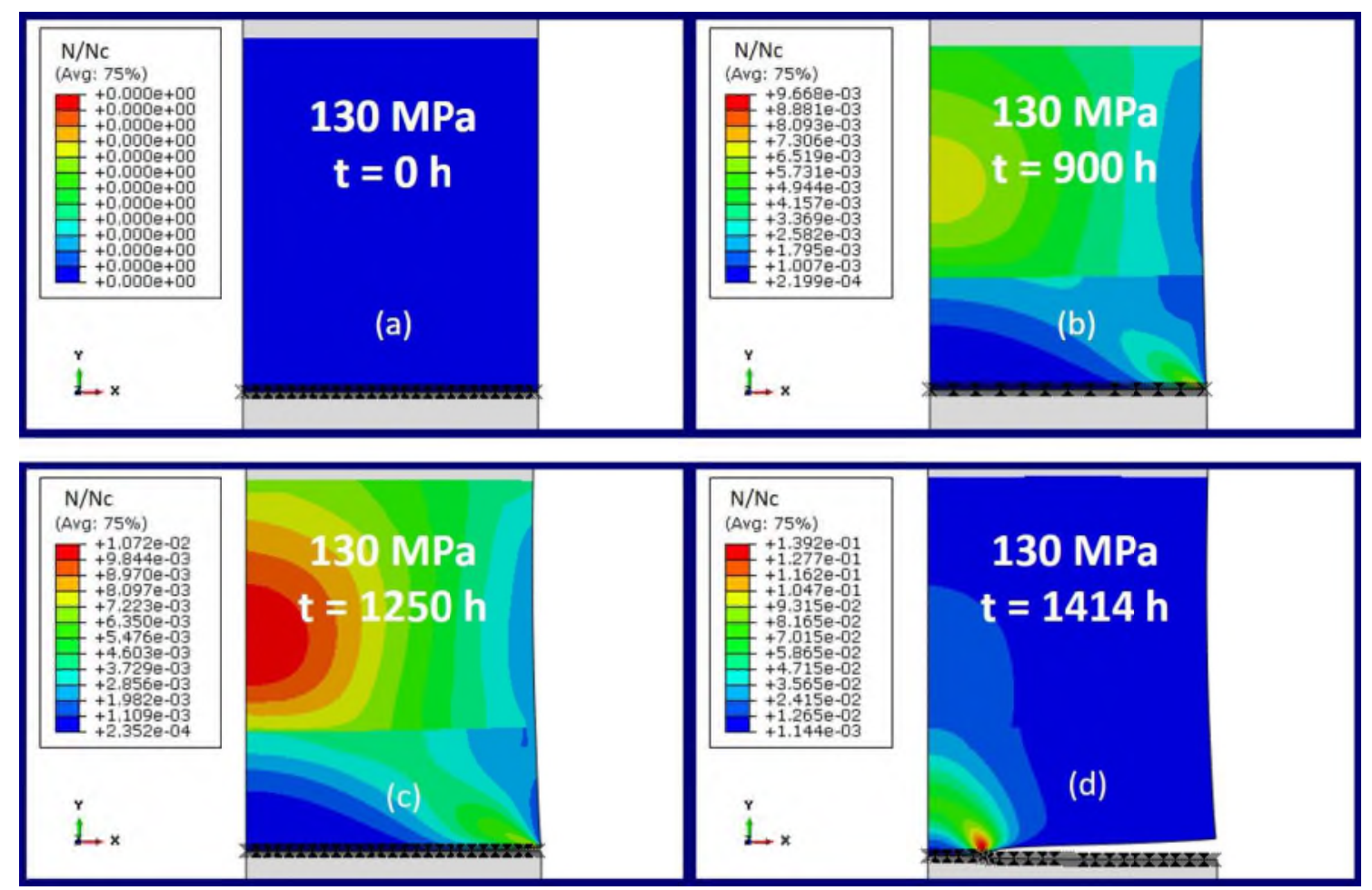

Fig. 9. Evolution of the solution dependent variable $N / N_{c}$ (number ratio of creep cavities Eq. 3) in HAZ of P22/Inco82 DMW system, subjected to a remote applied stress of $130 \mathrm{MPa}$, at different creep rupture stages: (a) before creep; (b) at crack initiation; (c) crack propagation and (d) final stage of creep rupture.

\subsection{Effect of change of material creep properties surrounding the dissimilar interface}

The comparison between P91/Inco and P22/Inco DMWs presented in section 4.1 in terms of crack initiation and propagation life highlights the significance of creep property mismatch between the two materials surrounding the dissimilar interface (Inconel and CGHAZ of the ferritic steel). Here a number of sensitivity studies are carried out, both in terms of physical properties and 
geometrical constraint. Firstly, in this section, we take P91/Inco82 DMWs and the same axisymmetric model in Fig. 4(b) as an example and further examine the effect of change of creep property mismatch on the creep rupture life. In practice, we only change the reference creep rate $\left(\dot{\varepsilon}_{0 H}\right.$ in Eq. 2 or $\dot{\varepsilon}_{0}$ in Eq. 1) but do not change the nature of the weldment configuration [8, 11, 12]. All other parameters remain unchanged. Four cases are conducted here:

CASE 1: increase $\dot{\varepsilon}_{0 H}$ by three orders of magnitude in P91 CGHAZ while keeping that of Inco82 constant;

CASE 2: decrease $\dot{\varepsilon}_{0 H}$ by three orders of magnitude in P91 CGHAZ while keeping that of Inco82 constant;

CASE 3: increase $\dot{\varepsilon}_{0}$ by three orders of magnitude in Inco82 while keeping that of P91 CGHAZ constant;

CASE 4: decrease $\dot{\varepsilon}_{0}$ by three orders of magnitude in Inco82 while keeping that of P91 CGHAZ constant.

The simulation results are shown in Fig. 10. Both data measured by Laha et al [3], Yamazaki et al [5] and Yamashita et al [6] as well as the original predicted result for P91/Inco82 DMWs are presented in the figure for comparison. The total creep rupture life is divided into crack initiation and propagation and is presented in Table 2 as ratios relative to the original values.

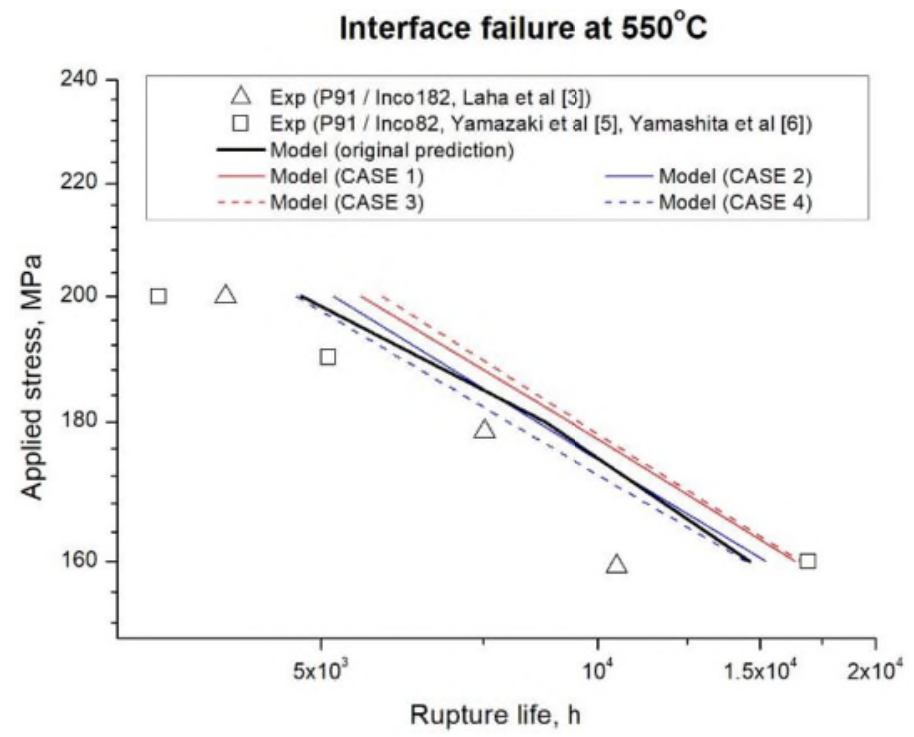

Fig. 10. Effect of change of reference creep rate in P91 CGHAZ and Inco82 on the creep rupture life of P91/Inco82 DMW system. Both data measured by Laha et al [3], Yamazaki et al [5] and Yamashita et al [6] as well as the original predicted result of P91/Inco82 DMWs are shown in the figure for a comparison.

Table 2. Simulated crack initiation and propagation life from CASE 1 to CASE 4 (ratios are relative to the original value)

\begin{tabular}{|c|c|c|c|c|c|c|c|c|}
\hline \multirow{2}{*}{$\begin{array}{c}\text { Applied } \\
\text { stresses (MPa) }\end{array}$} & \multicolumn{4}{|c|}{ Crack initiation } & \multicolumn{4}{c|}{ Crack propagation } \\
\cline { 2 - 9 } & CASE 1 & CASE 2 & CASE 3 & CASE 4 & CASE 1 & CASE 2 & CASE 3 & CASE 4 \\
\hline $\mathbf{2 0 0}$ & $\sim 1.23$ & $\sim 1.37$ & $\sim 1.58$ & $\sim 1.23$ & $\sim 0.90$ & $\sim 0.14$ & $\sim 0.05$ & $\sim 0.18$ \\
\hline $\mathbf{1 7 5}$ & $\sim 1.07$ & $\sim 1.20$ & $\sim 1.31$ & $\sim 1.12$ & $\sim 1.68$ & $\sim 0.20$ & $\sim 0.40$ & $\sim 0.22$ \\
\hline $\mathbf{1 6 0}$ & $\sim 1.08$ & $\sim 1.05$ & $\sim 1.14$ & $\sim 0.99$ & $\sim 4.00$ & $\sim 0.55$ & $\sim 1.00$ & $\sim 1.50$ \\
\hline
\end{tabular}

In general, it is observed that for all the cases the creep rupture life has not significantly changed, indicating that the total rupture life is insensitive to the change of reference creep rates of the materials surrounding the interface within the range of changes adopted in this study. There are however some important differences between the different sets of results. For example, an increase in the reference strain rate (CASE 1 and CASE 3) gives rise to $20 \%$ increase in the total life while a decrease (CASE 2 and CASE 4) results in a similar life to the original at each stress. Further, the detailed contribution to the total life from crack initiation and propagation varies, as reflected in Table 2. The following two trends can be found in Table 2:

(1) A longer crack initiation life than the original is found in most CASES, which still takes the dominant part in the total rupture 
life compared with the propagation.

(2) As the applied stress decreases, a change of creep properties of the materials surrounding the interface tends to give a longer propagation life than the original.

Taking the applied stress of $160 \mathrm{MPa}$ as an example, the quantitative damage accumulation along the interface is presented in Fig. 11. A similar damage evolution process is found in CASE 2 (Fig. 11b) and CASE 4 (Fig. 11d) compared with the original (Fig. 6c), with a smooth distribution of damage along the interface. The crack initiation life is subtly enhanced in CASE 2 while that in CASE 4 remains almost unchanged. Interestingly, a different damage evolution process is found in CASE 1 (Fig. 11a) and CASE 3 (Fig. 11c) compared with the original (Fig. 6c), with a rather uniform damage accumulation in the interior region of the interface. CASE 1 even demonstrates a significantly sharper transition compared with other CASES close to the free edge at the outside surface, which is observed to gradually decrease with time. Here we attempt to explain these features in terms of two major effects that occur as a result of the change in creep properties i.e.: a) change in the creep mismatch between the two materials surrounding the interface as shown in Fig. 3(c); and b) change in the relaxation rate of the normal traction close to the free edge.

A decrease of the reference creep rate in the P91 CGHAZ (CASE 2) reduces both the relaxation rate of the normal traction close to the free edge and the creep mismatch, with the former potentially increasing and the latter decreasing the damage accumulation rate at the free edge, in terms of Eq. (6). The later crack initiation in CASE 2 (Fig. 11b) than the original is thus a combined effect but is dominated by the reduced creep mismatch. A decrease of the reference creep rate of Inco82 (CASE 4) gives rise to very limited change in the traction relaxation and the creep mismatch (as the P91 CGHAZ creeps faster than Inco82 at the considered stresses). As a result, the damage evolution resembles that of the original (Fig. 11d). An increase in the reference creep rate of the P91 CGHAZ (CASE 1) enhances both the relaxation rate of the normal traction and the creep mismatch, which is the opposite of CASE 2. The faster damage growth along the interior region of the interface (Fig. 11a) is a direct result of the two effects, where the normal traction at the interior region increases by the enhanced traction relaxation around the free edge (the average normal traction must be in equilibrium with the remote applied stress). Also due to the enhanced relaxation of the traction in the region with greater damage around the free edge, the region does not grow into the interior, which allows the damage to grow uniformly. The even later crack initiation in CASE 1 than that in CASE 2 is again a combined effect, but is dominated more by the enhanced traction relaxation. Finally, an increase in reference creep rate in Inco82 (CASE 3) reduces the creep mismatch but enhances the traction relaxation rate, both of which can reduce the damage growth rate, as reflected in the longest crack initiation life in Fig. 11(c). The damage evolution in the interior region is faster than CASEs 2 and 4 and also the original situation, as a result of the enhanced traction relaxation (equilibrium effect as mentioned above), but is slower than CASE 1 because of a reduced creep mismatch. 
Damage accumulation along interface (Reference creep rate increase by $10^{3}$ in CGHAZ)

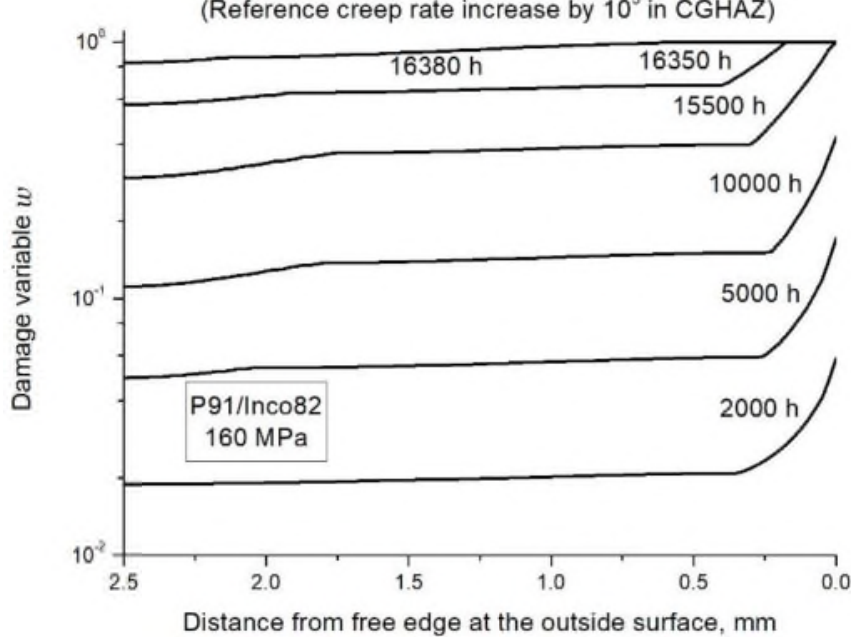

(a)

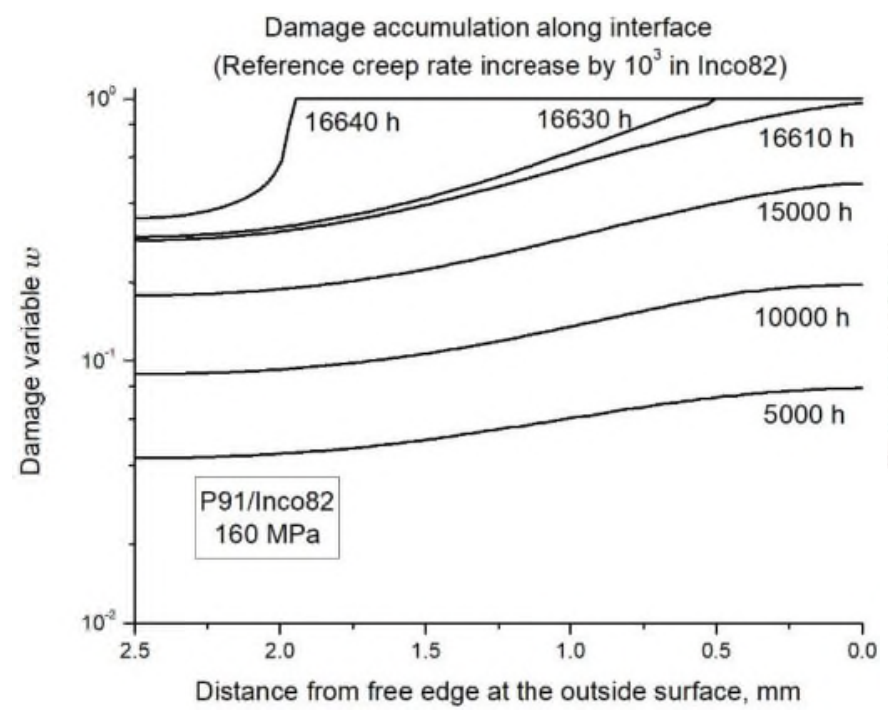

(c)

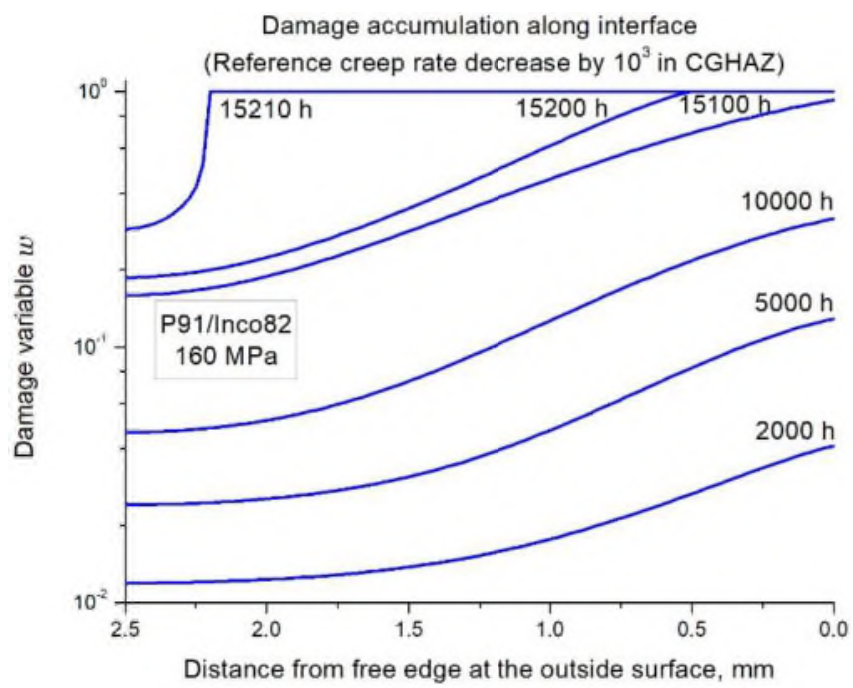

(b)

Damage accumulation along interface (Reference creep rate decrease by $10^{3}$ in Inco82)

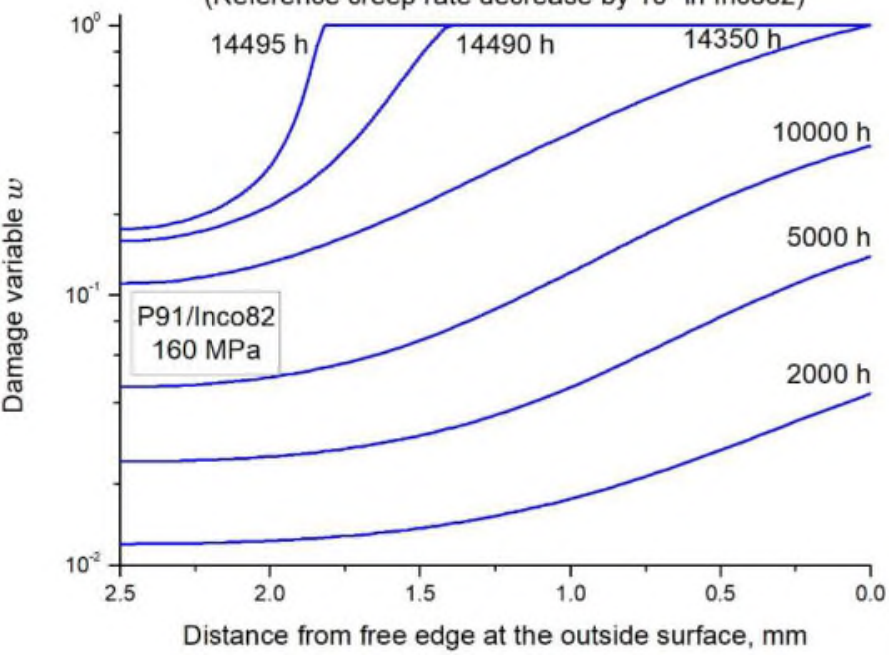

(d)

Fig. 11. Comparison of damage accumulation along the interface of P91/Inco82 DMW system at 160MPa in (a) CASE 1 (increase of reference creep rate in P91 CGHAZ); (b) CASE 2 (decrease of reference creep rate in P91 CGHAZ); (c) CASE 3 (increase of reference creep rate in Inco82) and (d) CASE 4 (decrease of reference creep rate in Inco82).

\subsection{Effect of different geometric constraints (plane stress \& plane strain)}

In this section, we further explore how the change of geometric constraint influences the interface creep rupture life, taking P91/Inco82 DMW system as an example. Here we establish a two-dimensional plate model in ABAQUS (Fig. 12) with the gauge length and thickness of HAZ exactly the same as that of the axisymmetric solid cylinder model shown in Fig. 4(b), similar to the structure in our previous work [16] but with the HAZ incorporated. Note that the width of the plate equals the diameter of the solid cylinder rod. The mesh of the plate model follows the criteria evaluated before in section 4.1. Plane stress (CPS4) and plane strain (CPE4) elements in ABAQUS are chosen for the continuum materials for a comparison. All parameters remain the same, except that the numerical integration area of each interface element is modified in the UEL from axisymmetric problems to plane problems, i.e. $\int 2 \pi r d r d \theta \rightarrow \int d x d y[38]$. 


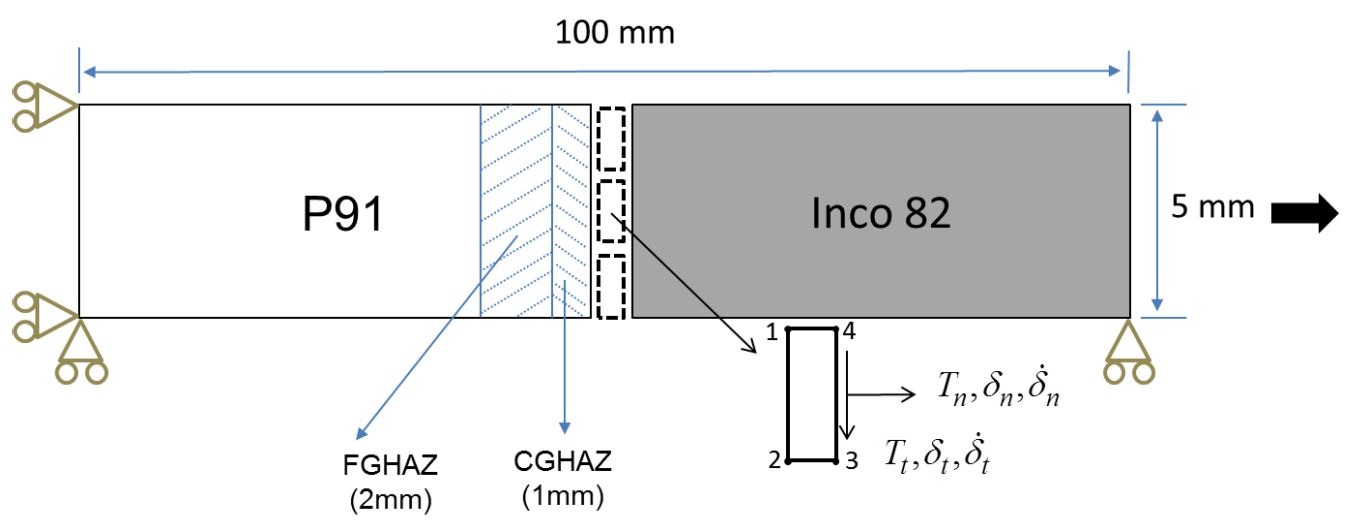

Fig. 12. Schematic of two-dimensional plate models of P91/Inco82 DMW system subject to uniaxial creep loading.

The predicted results of total creep rupture life for the plate models at the aforementioned same stress levels are shown in Fig. 13. Results using the data measured by Laha et al [3], Yamazaki et al [5] and Yamashita et al [6] are compared with the original predicted results for P91/Inco82 DMWs using an axisymmetric solid cylinder model. The predicted total creep rupture life for both plane stress and plane strain models is found to be shorter than the axisymmetric model, but still within the scatter of the experimental data. The plane strain model is further observed to be more creep-resistant than the plane stress model and agrees better with the actual axisymmetric model at the applied stress levels used in these simulations. The crack initiation and propagation life at each applied stress normalized by the life for the original axisymmetric solid cylinder model are tabulated in Table 3. Both plane problems exhibit a majority of their rupture life spent initiating a crack, and tend to give rise to a longer propagation life than the original as the applied stress level decreases, which are consistent with the previous findings in the axisymmetric problem, but the ratio between crack initiation and propagation varies. At each applied stress, plane stress is found to exhibit a shorter period of crack initiation but longer propagation life than plane strain.

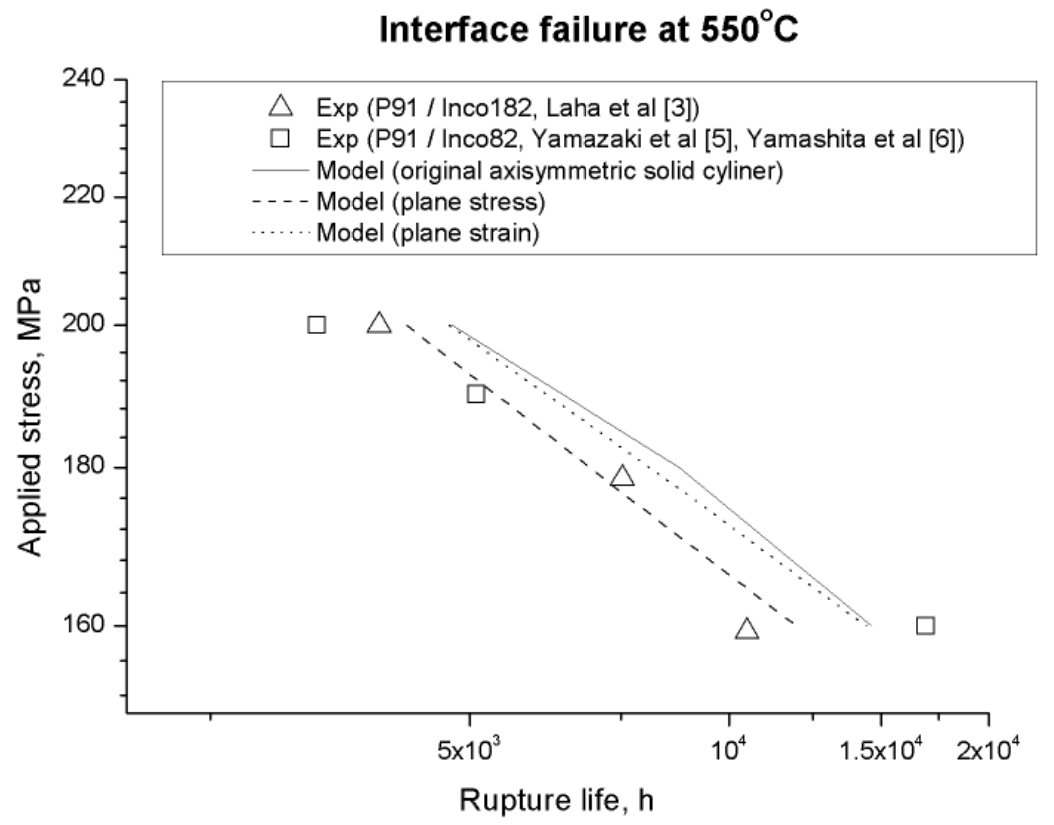

Fig. 13. Simulated (plane model) creep rupture life of P91/Inco82 DMW system. Data of P91/Inco182 tested by Laha et al [3], Yamazaki et al [5] and Yamashita et al [6] are shown, together with the original predicted result using the axisymmetric model.

Table 3. Simulated crack initiation and propagation life of plane stress / strain problems (ratios are relative to the original value) 


\begin{tabular}{|c|c|c|c|c|}
\hline \multirow{2}{*}{ Applied stresses (MPa) } & \multicolumn{2}{|c|}{ Crack initiation } & \multicolumn{2}{c|}{ Crack propagation } \\
\cline { 2 - 5 } & Plane stress & Plane strain & Plane stress & Plane strain \\
\hline $\mathbf{2 0 0}$ & $\sim 0.90$ & $\sim 0.99$ & $\sim 0.90$ & $\sim 0.90$ \\
\hline $\mathbf{1 7 5}$ & $\sim 0.73$ & $\sim 0.93$ & $\sim 1.60$ & $\sim 1.44$ \\
\hline $\mathbf{1 6 0}$ & $\sim 0.56$ & $\sim 0.85$ & $\sim 20.0$ & $\sim 11.0$ \\
\hline
\end{tabular}

Taking the applied stress $160 \mathrm{MPa}$ as an example, the distribution of axial stress around the interface and the evolution of the state variable $N / N_{c}$ in the HAZ at different creep rupture stages are shown in Figs. 14-15 (plane stress) and Figs. 16-17 (plane strain), illustrating the crack initiation and propagation stages. The stress legend scales in Figs. 14 and 16 have been unified for comparison. The main observations are listed below.

(1) Cracks initiate at both edges when the interface meets the free surface in the plane problems, which is consistent with the axisymmetric solid cylinder problem where the crack initiates from the outer surface around the entire circumference.

(2) Asymmetric crack propagation is observed, which results from numerical perturbation in the computation. A small perturbation may accumulate and influence subsequent computations. This would result in asymmetric evolution of damage in the interface elements and ultimately leads to a single predominant crack propagation path.

(3) Before initiation of any crack, a stress singularity where the interface meets the free surface is similar in both plane problems (Fig. 14 a \& Fig. 16 a). However, the traction concentration relaxes faster in plane strain at the interface than that in plane stress, leading to a much delayed crack initiation in plane strain. Once a crack initiates, the more constraint in plane strain leads to higher triaxiality [8] ( twice that in plane stress, not shown here) at the crack tip surrounding the interface, thus resulting in higher stresses at the crack tip than for plane stress (Fig. $14 \mathrm{c} \& d$, Fig. $16 \mathrm{c} \& d$ ). As a result, the creep crack growth rate is faster in plane strain along an already heavily damaged interface, giving rise to a much shorter crack propagation life than plane stress.

(4) Localized cavity nucleation can be seen at the crack tip as the crack propagates towards the center. A potential for diversion of the crack path into the HAZ due to the formation of a large number of grain-sized cavities can also be seen, although this is less pronounced than in the axisymmetric model (Fig. 8).

These results and comparisons demonstrate that the change of geometric constraints of the problem can affect the detailed combination of crack initiation and propagation life at the dissimilar interface, but would not change significantly the total creep rupture life and nature of the creep rupture process. 

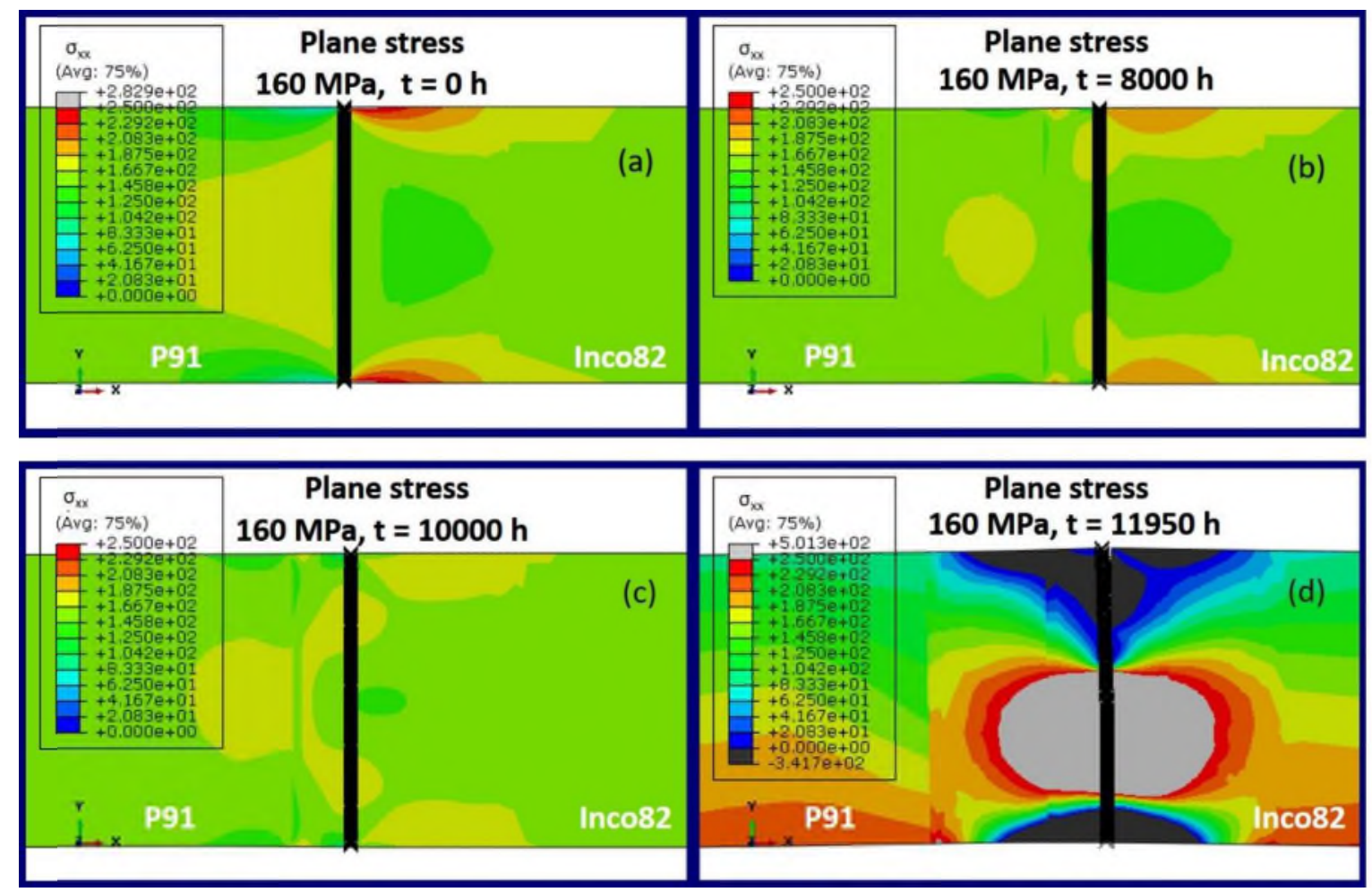

Fig. 14. Plane stress model: Axial stress distribution around interface of P91/Inco82 DMW system subjected to a remote applied stress of $\mathbf{1 6 0}$ MPa at different creep rupture stages: (a) before creep; (b) at crack initiation; (c) crack propagation and (d) final stage of creep rupture.
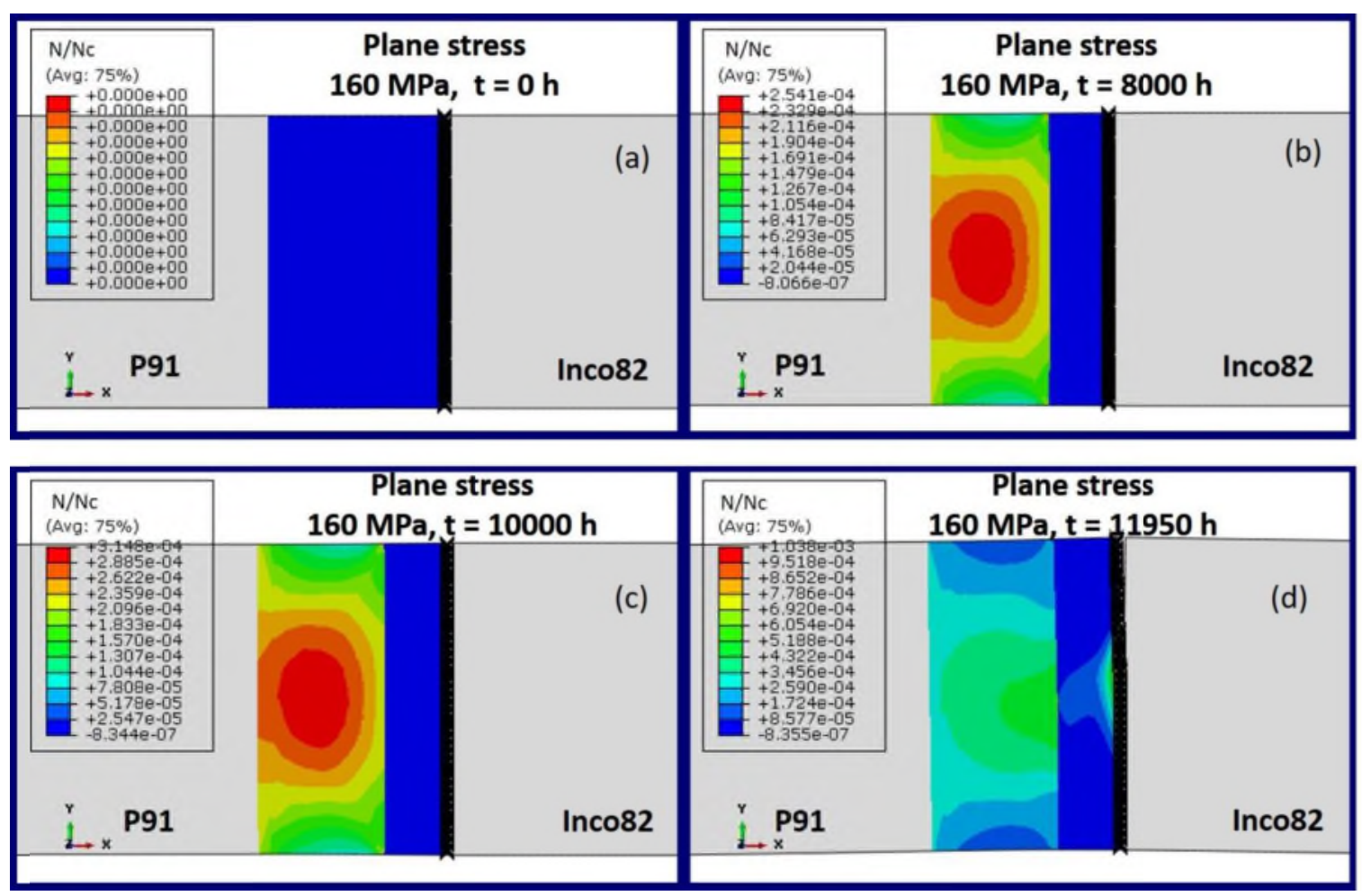

Fig. 15. Plane stress model: Evolution of the state variable $N / N_{c}$ in HAZ of P91/Inco82 DMW system, subjected to a remote applied stress of 160 MPa, at different creep rupture stages: (a) before creep; (b) at crack initiation; (c) crack propagation and (d) final stage of creep rupture. 

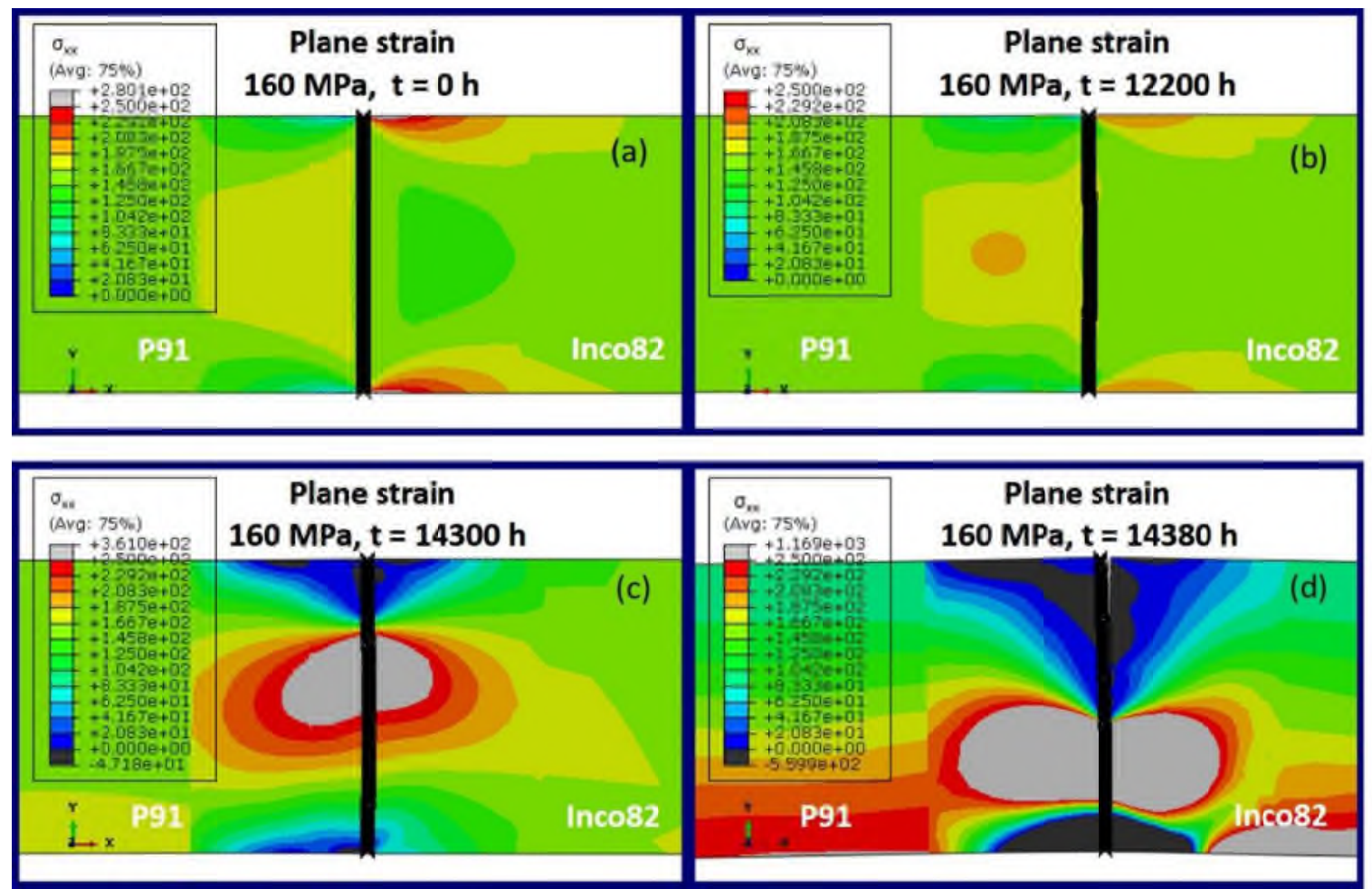

Fig. 16. Plane strain model: Axial stress distribution around interface of P91/Inco82 DMW system subjected to a remote applied stress of $\mathbf{1 6 0}$ MPa at different creep rupture stages: (a) before creep; (b) at crack initiation; (c) crack propagation and (d) final stage of creep rupture.
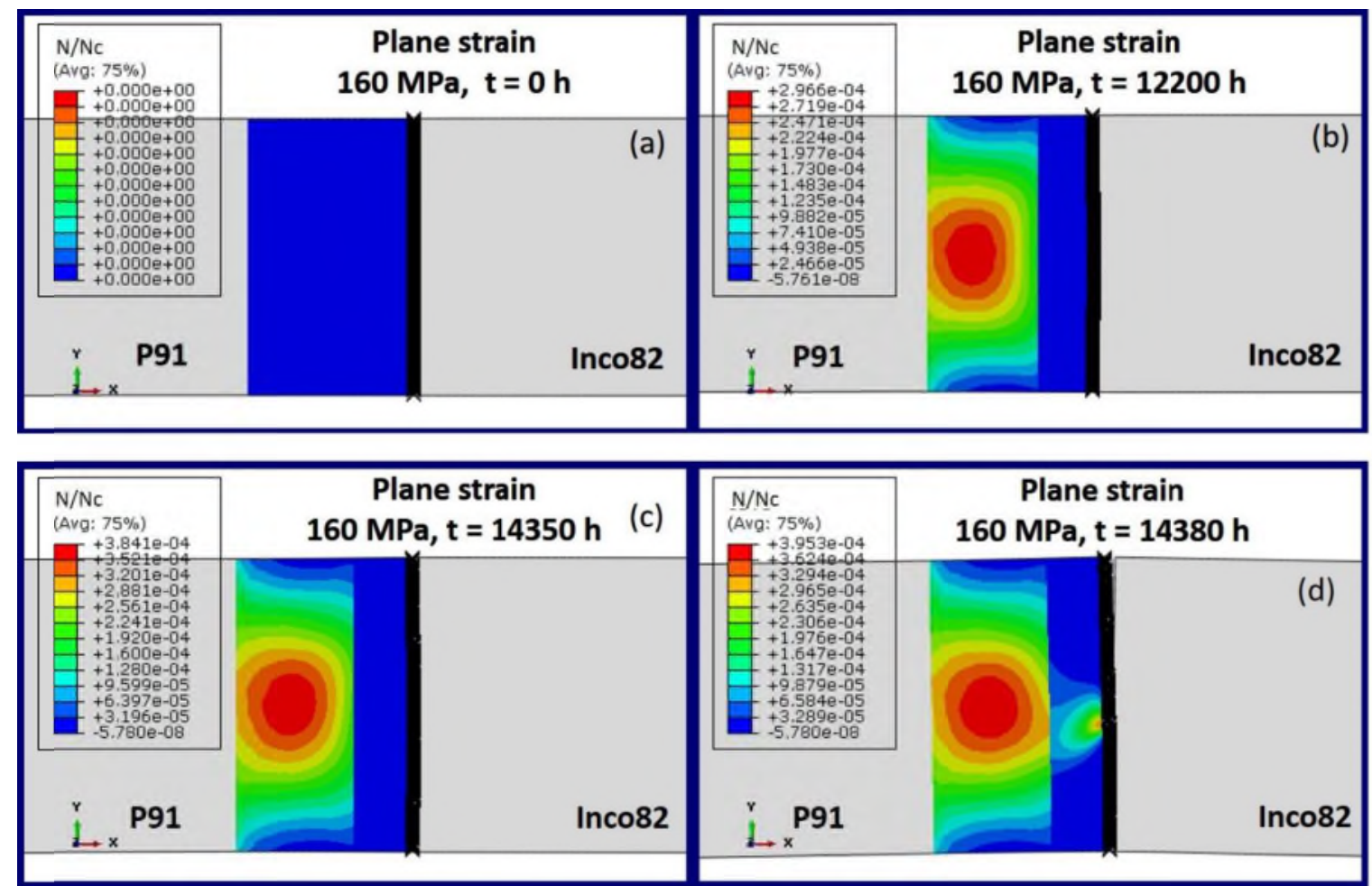

Fig. 17. Plane strain model: Evolution of the state variable $N / N_{c}$ in HAZ of P91/Inco82 DMW system, subjected to a remote applied stress of 160 MPa, at different creep rupture stages: (a) before creep; (b) at crack initiation; (c) crack propagation and (d) final stage of creep rupture.

\section{Discussions and conclusion}

In this paper damage development along a dissimilar metal weld interface and the associated degradation of elastic-creep properties of the interface has been described using a simple Kachanov type damage variable $\omega$, empirically defined as the ratio between the normal creep separation and a prescribed critical separation (Eq. 5). Many factors can influence the growth of damage 
at the dissimilar interface. These include specimen geometrical constraint, and also, from the mechanical perspective, the load level at a given temperature, as well as the effect of mismatch in creep properties of the surrounding materials. These have been examined and discussed in section 4 and also in the creep crack growth literature in similar metal welded components containing an initial crack at different positions $[7,8,10,12]$. Physically, the separation at the interface leading up to failure is caused by the nucleation, growth and coalescence of cavities, thus $\omega$ is related to the area fraction of cavities in the plane of the interface. The development of micromechanical interface models for this process is explored elsewhere [14, 39]. It is further noted that, for dissimilar metal welds, the preferential sites of these cavities have been found to be largely provided by the elongated carbides that nucleate and grow at the interface throughout the life [3]. Cocks and Ashby [40] and Cocks and Ponter [41] have explored the relationship between micromechanical and empirical Kachanov models and demonstrate that if a single process dominates the rupture process, it is possible to map from one description to the other. Under these conditions we would expect the results presented here to provide a good description of the material response and an accurate representation of the development of damage in a welded component. In situations where multiple processes determine the rupture life, the material description may need to be further developed to provide a more elaborate multi-state variable micromechanical model in order to adequately capture the appropriate constitutive response.

To conclude, in this paper, we have adopted an empirical interface zone model with a Kachanov-type ductility exhaustion damage variable to simulate and evaluate the uniaxial creep rupture at $550^{\circ} \mathrm{C}$ due to failure at dissimilar weld interfaces of different P91/Inco and P22/Inco dissimilar metal weld (DMW) systems, as tested independently by Laha et al [3] and Yamazaki et al [5] and Yamashita et al [6]. An axisymmetric solid cylinder model is established in ABAQUS. Creep parameters have been calibrated against the available experimental interface failure data obtained by Laha et al [3]. The calibrated model has been further used in a number of sensitivity studies taking P91/Inco82 as an example, such as the effect of change in creep property mismatch and geometric constraint. The main findings are summarized below:

(1) Similarities and differences in the damage accumulation along the interface of different DMW systems can be largely attributed to the mismatch in creep properties of the continuum materials surrounding the dissimilar interface and the associated traction relaxation close to the free edge. A proper design for creep mismatch between weldment constituents can possibly enhance the creep rupture life.

(2) A major proportion of the creep failure life at a flat interface is taken by crack initiation. A wavy interface would be expected to enhance the ratio between crack propagation / initiation, as it can provide a more significant enhancement of stress where the interface meets the free surface and more positions along the irregular interface where the stress is enhanced, promoting the nucleation of internal cracks, and also a tortuous crack path, which could slow down the crack propagation.

(3) Diversion of the crack path into HAZ, i.e. Type VI failure mode, can be qualitatively captured by monitoring the damage (cavity) accumulation in the HAZ. However, such a diversion would not affect the total life, since it only occurs at the very late stage of creep rupture when the crack propagates very rapidly.

(4) A change in the geometric constraint may change the relationship between crack initiation and propagation, but does not affect significantly the total creep rupture life and general features of the creep rupture process in the DMWs described above.

The model presented here can be used to assess actual high-temperature DMW components at lower stress levels with longer creep life, as the failure at lower stress levels is still dominated by the interface failure mode (Fig. 2). The structure can be further extended to incorporate the detailed physics of damage accumulation by characterizing the evolution of carbides and cavities at the interface within the framework of a micromechanical description.

\section{Acknowledgement}

Jianan Hu and Alan Cocks would like to acknowledge the financial support of Mitsubishi Heavy Industries (MHI). 


\section{References}

[1] Mvola B, Kah P, Martikainen J. Dissimilar Ferrous Metal Welding Using Advanced Gas Metal Arc Welding Processes. Reviews on Advanced Materials Science. 2014;38:125-37.

[2] Clark JWG, McCartney DG, Saghafifar H, Shipway PH. Modelling Chemical and Microstructural Evolution across Dissimilar Interfaces in Power Plants. Proceedings of the Asme Power Conference, 2014, Vol 1. 2014.

[3] Laha K, Chandravathi KS, Parameswaran P, Goyal S, Mathew MD. A Comparison of Creep Rupture Strength of Ferritic/Austenitic Dissimilar Weld Joints of Different Grades of Cr-Mo Ferritic Steels. Metallurgical and Materials Transactions a-Physical Metallurgy and Materials Science. 2012;43A:1174-86.

[4] DuPont JN. Microstructural evolution and high temperature failure of ferritic to austenitic dissimilar welds. International Materials Reviews. 2012;57:208-34.

[5] Yamazaki M, Watanabe T, Hongo H, Tabuchi M. Creep rupture properties of welded joints of heat resistant steels. Journal of Power and Energy Systems. 2008;2:1140-9.

[6] Yamashita T, Takaya S, Nagae Y, Date S, Tomobe M. Factor analysis of interface failure mechanism for dissimilar weld joints. Japan Atomic Energy Agency (JAEA) internal report. 2014.

[7] Chen G, Wang GZ, Zhang JW, Xuan FZ, Tu ST. Effects of initial crack positions and load levels on creep failure behavior in P92 steel welded joint. Eng Fail Anal. 2015;47:56-66.

[8] Chen G, Wang GZ, Xuan FZ, Tu ST. Mismatch effect in creep properties on creep crack growth behavior in welded joints. Mater Design. 2014;63:600-8.

[9] Chen G, Wang GZ, Xuan FZ, Tu ST. Effects of HAZ widths on creep crack growth properties of welded joints. Weld World. 2015;59:851-60.

[10] Segle P, Andersson P, Samuelson A. Numerical investigation of creep crack growth in cross-weld CT specimens. Part I: influence of mismatch in creep deformation properties and notch tip location. Fatigue Fract Eng M. 2000;23:521-31.

[11] Tu ST, Yoon KB. The influence of material mismatch an the evaluation of time-dependent fracture mechanics parameters. Eng Fract Mech. 1999;64:765-80.

[12] Lee KH, Kim YJ, Yoon KB, Nikbin K, Dean D. Quantification of stress redistribution due to mismatch in creep properties in welded branch pipes. Fatigue Fract Eng M. 2010;33:238-51.

[13] Park K, Paulino GH. Computational implementation of the PPR potential-based cohesive model in ABAQUS: Educational perspective. Engineering Fracture Mechanics. 2012;93:239-62.

[14] Hu J, Elmukashfi E, Fukahori T, Igari T, Chuman Y, Cocks ACF. Effect of weld angle on the interface creep rupture life of dissimilar metal welded components. In preparation. 2017.

[15] Wen JF, Tu ST, Gao XL, Reddy JN. Simulations of creep crack growth in 316 stainless steel using a novel creep-damage model. Engineering Fracture Mechanics. 2013;98:169-84.

[16] Hu J, Fukahori T, Igari T, Chuman Y, Cocks ACF. An evaluation of creep rupture strength of ferritic/austenitic dissimilar weld interfaces using cohesive zone modelling. Procedia Structural Integrity. 2016;2:934-41.

[17] Li M, Barrett RA, Scully S, Harrison NM, Leen SB, O'Donoghue PE. Cyclic plasticity of welded P91 material for simple and complex power plant connections. Int J Fatigue. 2016;87:391-404.

[18] Kumar Y, Venugopal S, Sasikala G, Albert SK, Bhaduri AK. Study of creep crack growth in a modified 9Cr-1Mo steel weld metal and heat affected zone. Materials Science and Engineering a-Structural Materials Properties Microstructure and Processing. 2016;655:300-9.

[19] Mannan SL, Laha K. Creep behaviour of Cr-Mo steel weldments. Transactions of the Indian Institute of Metals. 1996;49:30320.

[20] Francis JA, Mazur W, Bhadeshia HKDH. Type IV cracking in ferritic power plant steels. Materials Science and Technology. 2006;22:1387-95.

[21] Igari T, Fukahori T, Kawashima F, Tokiyoshi T, Chuman Y, Komai N, et al. Micro-macro creep damage simulation for welded joints. Materials at High Temperatures. 2011;28:181-7.

[22] Laha K, Chandravathi KS, Parameswaran P, Rao KBS, Mannan SL. Characterization of microstructures across the heataffected zone of the modified 9Cr-1 Mo weld joint to understand its role in promoting type IV cracking. Metallurgical and Materials Transactions a-Physical Metallurgy and Materials Science. 2007;38A:58-68.

[23] Eggeler G, Ramteke A, Coleman M, Chew B, Peter G, Burblies A, et al. Analysis of Creep in a Welded P91 Pressure-Vessel. International Journal of Pressure Vessels and Piping. 1994;60:237-57.

[24] Lim R, Sauzay M, Dalle F, Tournie I, Bonnaillie P, Gourgues-Lorenzon AF. Modelling and experimental study of the tertiary creep stage of Grade 91 steel. International Journal of Fracture. 2011;169:213-28.

[25] Gonzalez D, Cocks ACF, Fukahori T, Igari T, Chuman Y. Creep failure of a P91 simulated heat affected zone material under multiaxial state of stress. Proceedings of 3rd International ECCC Conference on Creep \& Fracture. 2014.

[26] Masuyama F, Yamaguchi T. Creep damage initiation mechanism and life of martensitic structures. Proceedings of 51st Symposium on High-Temperature Strength of Materials. 2013:110-4.

[27] Dyson BF. Continuous Cavity Nucleation and Creep Fracture. Scripta Metallurgica. 1983;17:31-7.

[28] Kelly DA. Creep Cavitation Failure in Copper Subject to Biaxial Stress Systems at 250 Degreesc. Acta Metallurgica. 1975;23:1267-75.

[29] Vandergiessen E, Tvergaard V. Interaction of Cavitating Grain-Boundary Facets in Creeping Polycrystals. Mechanics of Materials. 1994; 17:47-69. 
[30] Fukahori T, Tokiyoshi T, Igari T, Chuman Y, Komai N. Prediction of Type IV creep failure of a seam-welded mod. 9Cr-1Mo elbow based on microscopic damage simulation. Materials at High Temperatures. 2017;34:194-207.

[31] Kawashima F, Igari T, Tokiyoshi T, Shiibashi A, Tada N. Micro-macro combined simulation of the damage progress in lowalloy steel welds subject to type IV creep failure. Jsme International Journal Series a-Solid Mechanics and Material Engineering. 2004;47:410-8.

[32] Chandravathi KS, Laha K, Rao KBS, Mannan SL. Microstructure and tensile properties of modified 9Cr-1Mo steel (grade 91). Materials Science and Technology. 2001;17:559-65.

[33] Park K, Paulino GH. Cohesive Zone Models: A Critical Review of Traction-Separation Relationships Across Fracture Surfaces. Applied Mechanics Reviews. 2011;64.

[34] Kachanov LM. Rupture time under creep conditions. International Journal of Fracture. 1999;97:Xi-Xviii.

[35] Rabotnov YN. Creep problems in structural members. Amsterdam: North-Holland. 1969.

[36] Elmukashfi E, Cocks ACF. A theoretical and computational framework for studying creep crack growth. Int J Fracture. 2017; IUTAM Baltimore.

[37] Yu CH, Huang CW, Chen CS, Gao YF, Hsueh CH. Effects of grain boundary heterogeneities on creep fracture studied by rate-dependent cohesive model. Engineering Fracture Mechanics. 2012;93:48-64.

[38] Chester SA, Di Leo CV, Anand L. A finite element implementation of a coupled diffusion-deformation theory for elastomeric gels. International Journal of Solids and Structures. 2015;52:1-18.

[39] Yalcinkaya T, Cocks ACF. Micromechanically motivated cohesive zone formulation for ductile fracture. ENUMATH2015. 2015.

[40] Cocks ACF, Ashby MF. Creep Fracture by Coupled Power-Law Creep and Diffusion under Multiaxial Stress. Metal Science. $1982 ; 16: 465-74$.

[41] Cocks ACF, Ponter ARS. Creep Deformation and Failure under Cyclic Thermal Loading. Nuclear Engineering and Design. $1989 ; 116: 363-87$.

\section{Appendix Computational scheme for cohesive zone models}

For the nonlinear constitutive traction-separation rate laws (Eqs. 4a and b) in cohesive zone models as described in the context for a creep rupture problem, update of normal and tangential tractions $\left(T_{n}, T_{t}\right)$ and damage variable $\left(\omega=\delta_{n}^{c r} / \delta_{c}\right)$ at each time increment is computed in UEL by Newton-Raphson iteration method. Expression of $\partial T / \partial \delta$ is also needed in order to comply with the global integration scheme for adjacent bulk elements. This appendix shows the detailed update process and how to compute $\partial T / \partial \delta$ from Eqs. (4a) and (4b).

For either normal or tangential direction, two unknowns ( $\Delta \delta^{c r}$ and $\Delta T$ ) are to be solved, thus two equations are required. At the beginning of each time increment, the initial values of $T_{n}, T_{t}, \delta_{n}^{c r}, \delta_{t}^{c r}$ are given from the last time increment, while the total separation increments $\Delta \delta_{n}, \Delta \delta_{t}$ are given from ABAQUS. The first equation for each direction can be derived from the creep component in Eqs. (4a) and (4b)

$$
\begin{array}{ll}
G_{n} & =\Delta \delta_{n}^{c r}-b_{n} \Delta t\left[\frac{T_{n}+\theta \Delta T_{n}}{1-\left(\delta_{n}^{c r}+\theta \Delta \delta_{n}^{c r}\right) / \delta_{c}}\right]^{m}=0 \\
G_{t} & =\Delta \delta_{t}^{c r}-b_{t} \Delta t\left[\frac{T_{t}+\theta \Delta T_{t}}{1-\left(\delta_{n}^{c r}+\theta \Delta \delta_{n}^{c r}\right) / \delta_{c}}\right]^{m}=0
\end{array}
$$

where the rates of $T_{n}, T_{t}$ and $\delta_{n}^{c r}$ over the time increment $\Delta t$ are determined at an instant $\theta \Delta t . \theta$ is an integration constant. In the simulations presented here a value of $\theta=0.5$ was chosen to ensure that the solution is unconditionally stable. The second equation for each direction can be derived from the elastic component in Eqs. (4a) and (4b), noting that 


$$
\begin{array}{ll}
T_{n}=\frac{1-\delta_{n}^{c r} / \delta_{c}}{a_{n}}\left(\delta_{n}-\delta_{n}^{c r}\right) & T_{n}+\Delta T_{n}=\frac{1-\left(\delta_{n}^{c r}+\Delta \delta_{n}^{c r}\right) / \delta_{c}}{a_{n}}\left[\left(\delta_{n}-\delta_{n}^{c r}\right)+\left(\Delta \delta_{n}-\Delta \delta_{n}^{c r}\right)\right] \\
T_{t}=\frac{1-\delta_{n}^{c r} / \delta_{c}}{a_{t}}\left(\delta_{t}-\delta_{t}^{c r}\right) & T_{t}+\Delta T_{t}=\frac{1-\left(\delta_{n}^{c r}+\Delta \delta_{n}^{c r}\right) / \delta_{c}}{a_{t}}\left[\left(\delta_{t}-\delta_{t}^{c r}\right)+\left(\Delta \delta_{t}-\Delta \delta_{t}^{c r}\right)\right]
\end{array}
$$

Therefore

$$
\begin{aligned}
H_{n} & =\frac{1-\left(\delta_{n}^{c r}+\Delta \delta_{n}^{c r}\right) / \delta_{c}}{a_{n}}\left[\left(\delta_{n}-\delta_{n}^{c r}\right)+\left(\Delta \delta_{n}-\Delta \delta_{n}^{c r}\right)\right]-\frac{1-\delta_{n}^{c r} / \delta_{c}}{a_{n}}\left(\delta_{n}-\delta_{n}^{c r}\right) \\
& =\Delta T_{n}-\frac{1-\left(\delta_{n}^{c r}+\Delta \delta_{n}^{c r}\right) / \delta_{c}}{a_{n}}\left(\Delta \delta_{n}-\Delta \delta_{n}^{c r}\right)+\frac{T_{n} \Delta \delta_{n}^{c r}}{\delta_{c}-\delta_{n}^{c r}}=0 \\
H_{t} & =\frac{1-\left(\delta_{n}^{c r}+\Delta \delta_{n}^{c r}\right) / \delta_{c}}{a_{t}}\left[\left(\delta_{t}-\delta_{t}^{c r}\right)+\left(\Delta \delta_{t}-\Delta \delta_{t}^{c r}\right)\right]-\frac{1-\delta_{n}^{c r} / \delta_{c}}{a_{t}}\left(\delta_{t}-\delta_{t}^{c r}\right) \\
& =\Delta T_{t}-\frac{1-\left(\delta_{n}^{c r}+\Delta \delta_{n}^{c r}\right) / \delta_{c}}{a_{t}}\left(\Delta \delta_{t}-\Delta \delta_{t}^{c r}\right)+\frac{T_{t} \Delta \delta_{n}^{c r}}{\delta_{c}-\delta_{n}^{c r}}=0
\end{aligned}
$$

For each direction, the two equations can be solved using Newton-Raphson iteration.

$$
\begin{aligned}
& G_{x}^{i+1}=G_{x}^{i}+\frac{\partial G_{x}^{i}}{\partial \Delta \delta_{x}^{c r}}\left(\delta \Delta \delta_{x}^{c r}\right)^{i}+\frac{\partial G_{x}^{i}}{\partial \Delta T_{x}}\left(\delta \Delta T_{x}\right)^{i}=0 \\
& H_{x}^{i+1}=H_{x}^{i}+\frac{\partial H_{x}^{i}}{\partial \Delta \delta_{x}^{c r}}\left(\delta \Delta \delta_{x}^{c r}\right)^{i}+\frac{\partial H_{x}^{i}}{\partial \Delta T_{x}}\left(\delta \Delta T_{x}\right)^{i}=0
\end{aligned}
$$

The subscript " $x$ " is " $n$ " for normal direction and " $t$ " for tangential direction. After $i+1$ iterations within the increment the values of $\left(\Delta \delta_{x}^{c r}, \Delta T_{x}\right)^{i+1}$ are given by

$$
\left\{\begin{array}{c}
\Delta \delta_{x}^{c r} \\
\Delta T_{x}
\end{array}\right\}^{i+1}=\left\{\begin{array}{c}
\Delta \delta_{x}^{c r} \\
\Delta T_{x}
\end{array}\right\}^{i}-\left[\begin{array}{cc}
\frac{\partial G_{x}^{i}}{\partial \Delta \delta_{x}^{c r}} & \frac{\partial G_{x}^{i}}{\partial \Delta T_{x}} \\
\frac{\partial H_{x}^{i}}{\partial \Delta \delta_{x}^{c r}} & \frac{\partial H_{x}^{i}}{\partial \Delta T_{x}}
\end{array}\right]^{-1}\left\{\begin{array}{l}
G_{x}^{i} \\
H_{x}^{i}
\end{array}\right\}=\left\{\begin{array}{c}
\Delta \delta_{x}^{c r} \\
\Delta T_{x}
\end{array}\right\}-\left[J_{x}\right]\left\{\begin{array}{l}
G_{x}^{i} \\
H_{x}^{i}
\end{array}\right\}
$$

where $\left[J_{x}\right]$ is the Jacobian matrix. The iteration process continues until a prescribed tolerance is reached for both $\Delta \delta_{x}^{c r}$ and $\Delta T_{x}$. As for $\partial T / \partial \delta$, it can be determined using a procedure as described below. Note that

$$
\frac{\partial T_{x}}{\partial \delta_{x}}=\frac{\partial \Delta T_{x}}{\partial \Delta \delta_{x}}
$$

Differentiating Eqs. (A1) and (A3) with respect to $\Delta \delta_{x}$ then gives

$$
\left[\begin{array}{ll}
\frac{\partial G_{x}}{\partial \Delta \delta_{x}^{c r}} & \frac{\partial G_{x}}{\partial \Delta T_{x}} \\
\frac{\partial H_{x}}{\partial \Delta \delta_{x}^{c r}} & \frac{\partial H_{x}}{\partial \Delta T_{x}}
\end{array}\right] \cdot\left\{\begin{array}{l}
\frac{\partial \Delta \delta_{x}^{c r}}{\partial \Delta \delta_{x}} \\
\frac{\partial \Delta T_{x}}{\partial \Delta \delta_{x}}
\end{array}\right\}=\left[J_{x}\right]\left\{\begin{array}{l}
\frac{\partial \Delta \delta_{x}^{c r}}{\partial \Delta \delta_{x}} \\
\frac{\partial \Delta T_{x}}{\partial \Delta \delta_{x}}
\end{array}\right\}=\left[\begin{array}{c}
0 \\
\frac{1}{a_{x}}
\end{array}\right]
$$

Given the value of Jacobian matrix determined in the final iteration described above, $\partial T / \partial \delta$ can be then computed by Eq. (A7). 